\title{
SPARSE OPTIMAL CONTROL OF THE KdV-BURGERS EQUATION ON A BOUNDED DOMAIN*
}

\author{
ANNE-CELINE BOULANGER ${ }^{\dagger}$ AND PHILIP TRAUTMANN ${ }^{\ddagger}$
}

\begin{abstract}
In this work we consider measure-valued optimal control problems involving the $\mathrm{KdV}$-Burgers (KdVB) equation as a state equation. These optimal control problems are motivated by an inverse problem and a control problem involving the flow of water in a channel over topography. Well-posedness of the optimal control problem is established which involves the investigation of the KdVB equation for irregular source terms. Moreover optimality conditions for the control problem are derived. Efficient numerical schemes based on spectral methods are proposed for the state and adjoint equation, as well as adequate optimization methods. The theoretical results are illustrated by two numerical examples.
\end{abstract}

Key words. sparse optimal control, Korteweg-de Vries equation, inverse problem, spectral methods

AMS subject classifications. 35Q93, 49J20, 65M70, 74J30

DOI. $10.1137 / 15 \mathrm{M} 1020745$

1. Introduction. The KdV equation first appeared in 1895 in the context of water waves; cf. [29]. It is designed to model the evolution of long water waves in a channel of rectangular cross section when the effects of nonlinearity and dispersion balance. This phenomenon gives rise to the so-called soliton, a wave traveling at constant speed without losing its shape. This equation has been theoretically widely studied: much work has been devoted to the derivation of the equation from Euler equations, e.g., $[47,19,51]$, but also to the proof of its well-posedness in various contexts; cf. [40, 28, 8] (periodic domain, on the real line, bounded domain), and to their controllability, see $[45,25,20,15]$. One application of the KdV equation, which is of particular interest for us, is the modeling of a flow in a narrow channel over an obstacle; cf. [39, 47, 48]. In that case, a source term is added to the right-hand side of the $\mathrm{KdV}$ equation, which represents the spatial derivative of the topography under the flow. The resulting equation is called the forced KdV equation. More generally, we consider the forced KdV-Burgers (KdVB) equation, which describes the viscous flow over a topography. This is done on the one hand to include viscosity and on the other hand for mathematical reasons. In this paper we propose a framework which can be used for two kinds of problems regarding the KdVB equation. We investigate an inverse problem in which we intend to reconstruct a time varying bottom topography, e.g., the locations of bumps with time-dependent height on the bottom of the channel, from noisy observations of the generated wave patterns. Second, we consider a control problem in which we want to create a certain desired flow by changing the topography under the flow. In this article, we consider a topography which is piecewise constant and has jumps with time varying heights. Thus its derivative is a linear combination of

* Received by the editors May 12, 2015; accepted for publication (in revised form) July 17, 2017; published electronically November 21, 2017.

http://www.siam.org/journals/sicon/55-6/M102074.html

Funding: The authors were supported by the International Research Training Group IGDK1754, funded by the DFG and FWF.

${ }^{\dagger}$ Chair of Optimal Control, Technische Universität München, 85748 Garching bei München, Germany (boulanger@ma.tum.de).

${ }^{\ddagger}$ Institute for Mathematics and Scientific Computing, University of Graz, A-8010 Graz, Austria (philip.trautmann@uni-graz.at). 
Dirac measures with time-independent positions $x_{i}$ and time-dependent magnitudes $u_{i}$, i.e.,

$$
\sum_{i=1}^{N} u_{i}(t) \delta_{x_{i}}(x)
$$

Then the control problem consist of finding the optimal positions and optimal timedepending heights of the jumps given a desired water flow $z$. In the inverse problem, we reconstruct the positions and time-dependent heights of the jumps given noisy measurements of the flow patterns $z$. As pointed out in [31] and [32] the space of vector measures $\mathcal{M}\left(\Omega_{c}, L^{2}(I)\right)$ with values in $L^{2}(I)$ contains sources of the form (1.1) and the use of its norm $\|\cdot\|_{\mathcal{M}\left(\Omega_{c}, L^{2}(I)\right)}$ as the control cost functional enhances optimal controls with a small spatial support, i.e., controls of the form (1.1). In this perspective, we follow the path introduced in $[31,32]$ and introduce a measurevalued optimal control problem posed in the space $\mathcal{M}\left(\Omega_{c}, L^{2}(I)\right)$ for the approximative solution of the mentioned optimal control and inverse problem. In particular, we focus on the optimal control problem

$$
\begin{array}{r}
\min _{u \in \mathcal{M}\left(\Omega_{c}, L^{2}(I)\right), y \in Y} J(y, u)=\frac{1}{2}\left(\left\|\chi_{\Omega_{o}} y-z_{1}\right\|_{L^{2}\left(I \times \Omega_{o}\right)}^{2}+\left\|\chi_{\Omega_{o}} y(T)-z_{2}\right\|_{L^{2}\left(\Omega_{o}\right)}^{2}\right) \\
+\alpha\|u\|_{\mathcal{M}\left(\Omega_{c}, L^{2}(I)\right)}
\end{array}
$$

with $\left(z_{1}, z_{2}\right) \in L^{2}\left(I \times \Omega_{o}\right) \times L^{2}\left(\Omega_{o}\right)$, where $y \in Y$ (the space $Y$ will be defined later on) is the solution of the KdVB equation with homogenous Dirichlet and Neumann boundary conditions on $\Omega=(0, L)$ :

$$
\left\{\begin{array}{l}
\partial_{t} y+\partial_{x} y+\partial_{x x x} y+y \partial_{x} y-\gamma \partial_{x x} y=u \text { in } I \times \Omega, \\
y(\cdot, 0)=y(\cdot, L)=\partial_{x} y(\cdot, L)=0 \text { in } I \\
y(0, \cdot)=y_{0}(\cdot) \text { in } \Omega .
\end{array}\right.
$$

The control acts on the control domain $\Omega_{c} \subseteq \Omega$. The state variable $y$ is tracked on the observation domain $\Omega_{o} \subseteq \Omega$. The parameter $\alpha>0$ is called control cost parameter and $\gamma \geq 0$ viscosity parameter. Other boundary conditions, for example, periodic ones, e.g., [7], or exterior ones, e.g., [4], can be also prescribed in (1.2a)-(1.2c) instead of Dirichlet and Neumann boundary conditions. It appears not to be straightforward to extend our analysis to these boundary conditions, since, for example, compact embeddings are not available on unbounded domains. Similar types of measure-valued control problems have already been studied in the case of linear elliptic and parabolic equations; see $[44,17,10,18]$ and $[11,14,13,12]$. Our problem is connected to [27], where the authors use the control cost functional

$$
u \mapsto \alpha\|u\|_{L^{1}\left(\Omega_{c}, L^{2}(I)\right)}+\frac{\varepsilon}{2}\|u\|_{L^{2}\left(I \times \Omega_{c}\right)}^{2}
$$

in connection with parabolic optimal control problems. This control cost functional promotes sparsity patterns of the optimal control which are constant in time (directional sparsity, joint sparsity). Our problem setting is equivalent to theirs for $\varepsilon=0$. The mathematical challenges of this work are twofold: On the one hand we prove time-global well-posedness of the state equation in the presence of an irregular source term, and on the other hand sparse optimal control of a nonlinear dispersive partial differential equation is also a novel research topic. 
The time-global wellposedness of (1.2a)-(1.2c) for $\left(u, y_{0}\right) \in L^{2}\left(I, H^{-1}(\Omega)\right) \times L^{2}(\Omega)$ plays a very important role in the analysis of $(\mathcal{P})$. It is established in the nonviscous case $(\gamma=0)$ for small data $\left(u, y_{0}\right)$ and the viscous case $(\gamma>0)$ for general data. Based on these results concerning the state equation we establish the existence of an optimal control in two different scenarios, namely, in the nonviscous case $\gamma=0$ with additional norm constraints $\|u\|_{\mathcal{M}\left(\Omega_{c}, L^{2}(I)\right)} \leq \beta$ on the control and in the viscous case $\gamma>0$ for general controls. Then we derive necessary optimality conditions for $(\mathcal{P})$ in the nonviscous case with norm constraint on the control as well as in the viscous case for general controls. The derivation requires the analysis of the linearized KdVB equation and of the adjoint linearized KdVB equation. Therefore, we discuss their wellposedness and regularity properties. Additionally, we prove existence of controls in the nonviscous case $\gamma=0$ with general controls based on the nonreduced approach; cf. [35]. This approach requires an additional regularization term for the state $y$ in the cost functional $(\mathcal{P})$. Then we discuss the discretization of $(\mathcal{P})$ based on a PetrovGalerkin method in space and the Crank-Nikolson-leap-frog (CNLF) method in time for $(1.2 \mathrm{a})-(1.2 \mathrm{c})$. In particular, we derive a suitable discrete adjoint state equation. For the algorithmical solution of $(\mathcal{P})$ we consider the viscous and nonviscous case without norm constraints on the control. Our strategy is based on an additional $L^{2}$-regularization in $(\mathcal{P})$ and a continuation strategy in the regularization parameter. The $L^{2}$-regularized problems are solved by a trust-region semismooth Newton method. We conclude with some numerical examples on the KdVB equation, which include an inverse problem and a control problem.

This paper is organized as follows. In section 2 properties of the control space $\mathcal{M}\left(\Omega_{c}, L^{2}(I)\right)$ are discussed. Section 3 deals with the time-global wellposedness of $(1.2 \mathrm{a})-(1.2 \mathrm{c})$. In section 4 the existence of optimal controls is proved based on the state reduced approach. Section 5 is concerned with the derivation of necessary optimality conditions of $(\mathcal{P})$. In section 6 the existence of optimal controls in the nonviscous case $(\gamma=0)$ without additional control constraints based on nonreduced approach is shown. Finally, section 7 displays the numerical methods which are adopted for the solution of the state equation and of the optimization problem. The section is concluded with two numerical experiments.

2. Control space. In this section we introduce the control space $\mathcal{M}\left(\Omega_{c}, L^{2}(I)\right)$ and its properties. The control set $\Omega_{c}$ is any relatively closed subset of $\Omega$. Let $u: \mathcal{B}\left(\Omega_{c}\right) \rightarrow L^{2}(I)$ be a countably additive mapping on the Borel sets $\mathcal{B}\left(\Omega_{c}\right)$ of $\Omega_{c}$ with values in $L^{2}(I)$. For $u$ we denote by $|u| \in \mathcal{M}^{+}\left(\Omega_{c}\right)$ (positive regular Borel measure) the total variation measure defined by

$$
|u|(B)=\sup _{\pi} \sum_{E \in \pi}\|u(E)\|_{L^{2}(I)}
$$

where $\pi$ is the set of all disjoint partitions of $B \in \mathcal{B}\left(\Omega_{c}\right)$. The space

$$
\mathcal{M}\left(\Omega_{c}, L^{2}(I)\right)=\left\{u: \mathcal{B}\left(\Omega_{c}\right) \rightarrow L^{2}(I): \quad u \text { countably additive, }|u|\left(\Omega_{c}\right)<\infty\right\}
$$

is the space of vector measures with values in $L^{2}(I)$. Equipped with the norm

$$
\|u\|_{\mathcal{M}\left(\Omega_{c}, L^{2}(I)\right)}=|u|\left(\Omega_{c}\right)
$$

it is a Banach space. The support of $u$, respectively, of its total variation measure $|u|$, is defined by

$$
\operatorname{supp} u=\operatorname{supp}|u|=\Omega \backslash\left(\bigcup\left\{B \text { open in } \Omega_{c}|| u \mid(B)=0\right\}\right) .
$$


The vector measure $u$ possesses a Radon-Nikodym derivative (see [33])

$$
u^{\prime} \in L^{\infty}\left(\left(\Omega_{c},|u|\right), L^{2}(I)\right) \text { with }\left\|u^{\prime}(\cdot)\right\|_{L^{2}(I)} \equiv 1
$$

with respect to its total variation measure $|u|$. So $u$ can be represented in the following way:

$$
\mathrm{d} u=u^{\prime} \mathrm{d}|u| .
$$

Next we inx'troduce the space $\mathcal{C}\left(\Omega_{c}, L^{2}(I)\right)$ of vector-valued continuous functions $p: \bar{\Omega}_{c} \rightarrow L^{2}(I)$ with $\left.p\right|_{\partial \Omega \cap \bar{\Omega}_{c}}=0$. Equipped with the norm

$$
\|p\|_{\mathcal{C}\left(\Omega_{c}, L^{2}(I)\right)}=\max _{x \in \Omega_{c}}\|p(x, \cdot)\|_{L^{2}(I)}
$$

it is a separable Banach space. The dual space of $\mathcal{C}\left(\Omega_{c}, L^{2}(I)\right)$ can be characterized by $\mathcal{M}\left(\Omega_{c}, L^{2}(I)\right)$, i.e.,

$$
\mathcal{C}\left(\Omega_{c}, L^{2}(I)\right)^{*} \cong \mathcal{M}\left(\Omega_{c}, L^{2}(I)\right) .
$$

A proof is given in [26]. The duality pairing between $\mathcal{C}\left(\Omega_{c}, L^{2}(I)\right)$ and $\mathcal{M}\left(\Omega_{c}, L^{2}(I)\right)$ takes the form

$$
\langle u, p\rangle_{\mathcal{M}\left(\Omega_{c}, L^{2}(I)\right), \mathcal{C}\left(\Omega_{c}, L^{2}(I)\right)}=\int_{\Omega}\left(u^{\prime}, p\right)_{L^{2}(I)} \mathrm{d}|u| .
$$

Next we introduce the space $L^{2}\left(I, \mathcal{M}\left(\Omega_{c}\right)\right)$. It is the space of weakly-* measurable functions $u: I \rightarrow \mathcal{M}\left(\Omega_{c}\right)$ which satisfy

$$
\int_{0}^{T}\|u(t)\|_{\mathcal{M}\left(\Omega_{c}\right)}^{2} \mathrm{~d} t<\infty
$$

where $\mathcal{M}\left(\Omega_{c}\right)$ is the space of Borel measures on $\Omega_{c}$ and $\|\cdot\|_{\mathcal{M}\left(\Omega_{c}\right)}$ is the total variation norm in $\mathcal{M}\left(\Omega_{c}\right)$. Furthermore it can be identified with the dual space of $\mathcal{C}\left(\Omega_{c}\right)$ which is the space of continuous functions on $\bar{\Omega}_{c}$ with $\left.p\right|_{\partial \Omega \cap \bar{\Omega}_{c}}=0$. There holds

$$
\mathcal{M}\left(\Omega_{c}, L^{2}(I)\right) \hookrightarrow L^{2}\left(I, \mathcal{M}\left(\Omega_{c}\right)\right) .
$$

Since $d=1$ we have the embedding $H_{0}^{1}(\Omega) \hookrightarrow \mathcal{C}\left(\Omega_{c}\right)$. Thus there holds $L^{2}\left(I, H_{0}^{1}(\Omega)\right) \hookrightarrow$ $L^{2}\left(I, C\left(\Omega_{c}\right)\right)$ and by duality $L^{2}\left(I, \mathcal{M}\left(\Omega_{c}\right)\right) \hookrightarrow L^{2}\left(I, H^{-1}(\Omega)\right)$.

3. Well-posedness of the state equation. In this section we discuss the timeglobal well-posedness of the state equation for irregular sources from $L^{2}\left(I, H^{-1}(\Omega)\right)$ which includes $\mathcal{M}\left(\Omega_{c}, L^{2}(I)\right)$ according to the last section. For the proof of the time-global well-posedness we need to distinguish the viscous case $\gamma>0$ and the nonviscous case $\gamma=0$. In the nonviscous case we are restricted to small data whereas in the viscous case no such constraints on the data are necessary. First we present some necessary results concerning the linear KdVB equation.

3.1. Well-posedness of the linear KdVB equation for $\gamma \geq 0$. First we consider

$$
\left\{\begin{array}{l}
\partial_{t} y+\partial_{x} y+\partial_{x x x} y-\gamma \partial_{x x} y=f \text { in } I \times \Omega \\
y(\cdot, 0)=y(\cdot, L)=\partial_{x} y(\cdot, L)=0 \text { in } I \\
y(0, \cdot)=y_{0}(\cdot) \text { in } \Omega
\end{array}\right.
$$

Copyright (c) by SIAM. Unauthorized reproduction of this article is prohibited. 
and its dual counter part

$$
\left\{\begin{array}{l}
-\partial_{t} p-\partial_{x} p-\partial_{x x x} p-\gamma \partial_{x x} p=\phi \text { in } I \times \Omega, \\
p(\cdot, 0)=p(\cdot, L)=\partial_{x} p(\cdot, 0)=0 \text { in } I, \\
p(T, \cdot)=p_{T}(\cdot) \text { in } \Omega,
\end{array}\right.
$$

where $\gamma \geq 0$ is assumed. So we consider the the viscous and nonviscous case at the same time. We denote by $A: L^{2}(\Omega) \rightarrow L^{2}(\Omega)$ the linear differential operator

$$
A w=-\partial_{x x x} w-\partial_{x} w+\gamma \partial_{x x} w
$$

with the dense domain $\mathcal{D}(A) \subset L^{2}(\Omega)$ defined by

$$
\mathcal{D}(A)=\left\{w \in H^{3}(\Omega) \text { s.t. } w(0)=w(L)=\partial_{x} w(L)=0\right\} .
$$

The adjoint operator $A^{*}: L^{2}(\Omega) \rightarrow L^{2}(\Omega)$ is given by

$$
A^{*} w=\partial_{x x x} w+\partial_{x} w+\gamma \partial_{x x} w
$$

with the domain

$$
\mathcal{D}\left(A^{*}\right)=\left\{w \in H^{3}(\Omega) \text { s.t. } w(0)=w(L)=\partial_{x} w(0)=0\right\} .
$$

The operators $A$ and $A^{*}$ generate strongly continuous semigroups of contractions on $L^{2}(\Omega)$ denoted by $W(\cdot): L^{2}(\Omega) \rightarrow L^{2}(\Omega)$ and $W^{*}(\cdot): L^{2}(\Omega) \rightarrow L^{2}(\Omega)$. The reader is referred to [45] for a proof. In what follows, we will denote by $\mathcal{B}$ the Banach space $C\left(I, L^{2}(\Omega)\right) \cap L^{2}\left(I, H_{0}^{1}(\Omega)\right)$ endowed with the norm

$$
\|y\|_{\mathcal{B}}=\|y\|_{\mathcal{C}\left(\bar{I}, L^{2}(\Omega)\right)}+\|y\|_{L^{2}\left(I, H_{0}^{1}(\Omega)\right)}, \quad y \in \mathcal{B},
$$

where $\|\cdot\|_{H_{0}^{1}(\Omega)}=\left\|\partial_{x} \cdot\right\|_{L^{2}(\Omega)}$. Furthermore we introduce the Hilbert space

$$
\mathcal{V}=H_{0}^{2}(\Omega)=\left\{v \in H^{2}(\Omega) \cap H_{0}^{1}(\Omega): \partial_{x} v(0)=\partial_{x} v(L)=0\right\}
$$

endowed with the norm $\|\cdot\|_{\mathcal{V}}=\left\|\partial_{x x} \cdot\right\|_{L^{2}(\Omega)}$. Thus we have $\mathcal{V}^{*}=H^{-2}(\Omega)$. The following existence and uniqueness result can be found in [6, section 2].

Proposition 3.1. Let $\left(f, y_{0}\right) \in L^{1}\left(I, L^{2}(\Omega)\right) \times L^{2}(\Omega)$ and $\left(\phi, p_{T}\right) \in L^{1}\left(I, L^{2}(\Omega)\right) \times$ $L^{2}(\Omega)$. Then (3.1a)-(3.1c) have a unique (mild) solution $y \in \mathcal{B}$ which is given by

$$
y(t)=W(t) y_{0}+\int_{0}^{t} W(t-s) f(s) \mathrm{d} s \quad \forall t \in I,
$$

and there exists a constant $c>0$ independent of $y_{0}, f$, and $y$ such that

$$
\|y\|_{\mathcal{B}} \leq c\left(\|f\|_{L^{1}\left(I, L^{2}(\Omega)\right)}+\left\|y_{0}\right\|_{L^{2}(\Omega)}\right)
$$

holds. Furthermore (3.2a)-(3.2c) have a unique solution $p \in \mathcal{B}$ given by

$$
p(t)=W^{*}(T-t) p_{T}+\int_{t}^{T} W^{*}(s-t) \phi(s) \mathrm{d} s \quad \forall t \in I,
$$

and there exits a constant $c>0$ such that

$$
\|p\|_{\mathcal{B}} \leq c\left(\|\phi\|_{L^{1}\left(I, L^{2}(\Omega)\right)}+\left\|p_{T}\right\|_{L^{2}(\Omega)}\right)
$$

holds. 
Next we introduce a weak formulation of (3.1) for sources $f \in L^{2}\left(I, H^{-1}(\Omega)\right)$.

Definition 3.2. For $\left(f, y_{0}\right) \in L^{2}\left(I, H^{-1}(\Omega)\right) \times L^{2}(\Omega)$ a function $y \in C\left(\bar{I}, L^{2}(\Omega)\right)$ is called a weak solution of (3.1a)-(3.1c) if it satisfies the following equation:

$$
\int_{0}^{T}(y, \phi)_{L^{2}(\Omega)} \mathrm{d} t+\left(y(T), p_{T}\right)_{L^{2}(\Omega)}=\int_{0}^{T}\langle f, p\rangle_{H^{-1}(\Omega), H_{0}^{1}(\Omega)} \mathrm{d} t+\left(y_{0}, p(0)\right)_{L^{2}(\Omega)}
$$

for all $\left(\phi, p_{T}\right) \in L^{1}\left(I, L^{2}(\Omega)\right) \times L^{2}(\Omega)$, where $p=p\left(\phi, p_{T}\right) \in \mathcal{B}$ is the mild solution of $(3.2 \mathrm{a})-(3.2 \mathrm{c})$.

In order to show existence of a weak solution we utilize a strategy based on the approximation of the data.

Proposition 3.3. Let $\left(f, y_{0}\right) \in L^{2}\left(I, H^{-1}(\Omega)\right) \times L^{2}(\Omega)$. Then, there exists a unique weak solution $y \in \mathcal{B} \cap H^{1}\left(I, \mathcal{V}^{*}\right)$ of (3.1a)-(3.1c). Furthermore there exists a constant $C(T, L)>0$ such that the following estimate holds:

$$
\|y\|_{\mathcal{B}}+\left\|\partial_{t} y\right\|_{L^{2}\left(I, \mathcal{V}^{*}\right)} \leq C(T, L)\left(\left\|y_{0}\right\|_{L^{2}(\Omega)}+\|f\|_{L^{2}\left(I, H^{-1}(\Omega)\right)}\right) .
$$

Proof. We choose sequences

- $\left\{f_{n}\right\}_{n \in \mathbb{N}} \subset \mathcal{C}^{1}\left(\bar{I}, L^{2}(\Omega)\right)$ with $f_{n} \rightarrow f$ in $L^{2}\left(I, H^{-1}(\Omega)\right)$,

- $\left\{y_{0, n}\right\}_{n \in \mathbb{N}} \subset \mathcal{D}(A)$ with $y_{0, n} \rightarrow y_{0}$ in $L^{2}(\Omega)$,

which exist due to density. According to [3, Part 2, Proposition 3.3] there exists a unique classical solution

$$
y_{n} \in \mathcal{C}(\bar{I}, \mathcal{D}(A)) \cap \mathcal{C}^{1}\left(\bar{I}, L^{2}(\Omega)\right)
$$

of (3.1) for data $f_{n}$ and $y_{0, n}$ which satisfies the weak form (3.4). Furthermore it can be shown that $y_{n}$ satisfy the following estimate:

$$
\left\|y_{n}\right\|_{\mathcal{B}} \leq C(T, L)\left(\left\|y_{n, 0}\right\|_{L^{2}(\Omega)}+\left\|f_{n}\right\|_{L^{2}\left(I, H^{-1}(\Omega)\right)}\right) .
$$

For a proof see Appendix A.1. This estimate implies that $\left\{y_{n}\right\}_{n \in \mathbb{N}}$ is a Cauchy sequence in $\mathcal{B}$ and therefore there exists a $y \in \mathcal{B}$ which satisfies (3.4) with the data $\left(f, y_{0}\right)$. This means that $y$ is a weak solution of (3.1). Its uniqueness can be shown

using standard arguments. Furthermore we can pass to the limit in (3.6) and get the first part of (3.5). Next we choose any $\psi \in \mathcal{C}_{c}^{\infty}(I \times \Omega)$ and set $\phi=-\partial_{t} \psi-A^{*} \psi$ in (3.2). Therefore $\psi$ is the solution of (3.2a) $-(3.2 \mathrm{c})$, and it holds that

$$
\begin{aligned}
\int_{0}^{T}\left(y,-\partial_{t} \psi\right)_{L^{2}(\Omega)} \mathrm{d} t & =\int_{0}^{T}\left(y, A^{*} \psi\right)_{L^{2}(\Omega)}+\langle f, \psi\rangle_{H^{-1}(\Omega), H_{0}^{1}(\Omega)} \mathrm{d} t \\
& \leq C(T, L)\left(\|y\|_{L^{2}\left(I, H_{0}^{1}(\Omega)\right)}+\|f\|_{L^{2}\left(I, H^{-1}(\Omega)\right)}\right)\|\psi\|_{L^{2}(I, \mathcal{V})} .
\end{aligned}
$$

Due to the density of $\mathcal{C}_{c}^{\infty}(I \times \Omega)$ in $L^{2}(I, \mathcal{V})$, there holds $y \in H^{1}\left(I, \mathcal{V}^{*}\right)$ and

$$
\left\|\partial_{t} y\right\|_{L^{2}\left(I, \mathcal{V}^{*}\right)} \leq C(T, L)\left(\|f\|_{L^{2}\left(I, H^{-1}(\Omega)\right)}+\left\|y_{0}\right\|_{L^{2}(\Omega)}\right) .
$$

DeFinition 3.4. The operator

$$
\mathcal{L}: L^{2}\left(I, H^{-1}(\Omega)\right) \times L^{2}(\Omega) \rightarrow \mathcal{B},\left(f, y_{0}\right) \mapsto y,
$$

where $y$ is the weak solution of (3.1a)-(3.1c), is called solution operator of (3.1a)(3.1c). It is linear and bounded. 
3.2. Time-local well-posedness of the KdVB equation with $\gamma \geq 0$. We consider in this section the nonlinear KdVB equation (1.2a)-(1.2c) with sources from $f \in L^{2}\left(I, H^{-1}(\Omega)\right)$ and show its time-local wellposedness in the viscous and nonviscous case, i.e., $\gamma \geq 0$. First we introduce a suitable solution concept for the KdVB equation

$$
\left\{\begin{array}{l}
\partial_{t} y+\partial_{x} y+\partial_{x x x} y+y \partial_{x} y-\gamma \partial_{x x} y=f \text { in } I \times \Omega, \\
y(., 0)=y(., L)=\partial_{x} y(., L)=0 \text { in } I \\
y(0, .)=0 \text { in } \Omega
\end{array}\right.
$$

for sources from $L^{2}\left(I, H^{-1}(\Omega)\right)$.

Definition 3.5. For $\left(y_{0}, f\right) \in L^{2}(\Omega) \times L^{2}\left(I, H^{-1}(\Omega)\right)$ a function $y \in \mathcal{B}$ is called a weak solution of $(3.7 \mathrm{a})-(3.7 \mathrm{c})$ if it satisfies the fixed point equation

$$
y=\mathcal{L}\left(f-y \partial_{x} y, y_{0}\right)
$$

or in other words

(3.8)

$$
\int_{0}^{T}(y, \phi)_{L^{2}(\Omega)} \mathrm{d} t+\left(y(T), p_{T}\right)_{L^{2}(\Omega)}=\int_{0}^{T}\left\langle f-y \partial_{x} y, p\right\rangle_{H^{-1}(\Omega), H_{0}^{1}(\Omega)} \mathrm{d} t+\left(y_{0}, p(0)\right)_{L^{2}(\Omega)}
$$

for all $\left(\phi, p_{T}\right) \in L^{1}\left(I, L^{2}(\Omega)\right) \times L^{2}(\Omega)$, where $p\left(\phi, p_{T}\right) \in \mathcal{B}$ is the solution of $(3.2 \mathrm{a})$ (3.2c).

The last definition makes sense considering the next lemma, which is also needed for the proof of existence of a solution to (3.7a)-(3.7c).

Lemma 3.6. Let $T>0, y \in \mathcal{B}$, and $z \in \mathcal{B}$, and then there exists a $c>0$ independent of $T$ such that

$$
\left\|y \partial_{x} y-z \partial_{x} z\right\|_{L^{2}\left(I, H^{-1}(\Omega)\right)} \leq c T^{1 / 4}\|y+z\|_{\mathcal{B}}\|y-z\|_{\mathcal{B}} .
$$

Proof. The proof is provided in Appendix A.2 and is largely inspired by [24].

Let us define for an arbitrary $\theta \leq T$ the space

$$
\mathcal{B}_{\theta}=C\left([0, \theta], L^{2}(\Omega)\right) \cap L^{2}\left((0, \theta), H_{0}^{1}(\Omega)\right),
$$

endowed with the norm

$$
\|y\|_{\mathcal{B}_{\theta}}=\|y\|_{C\left([0, \theta], L^{2}(\Omega)\right)}+\|y\|_{L^{2}\left([0, \theta], H_{0}^{1}(\Omega)\right)} .
$$

Proposition 3.7. For any $f \in L^{2}\left(I, H^{-1}(\Omega)\right)$ and $y_{0} \in L^{2}(\Omega)$, there exists a $T^{*} \in[0, T]$ depending on $\|f\|_{L^{2}\left(I, H^{-1}(\Omega)\right)}$ and $\left\|y_{0}\right\|_{L^{2}(\Omega)}$ such that the system (3.7a)(3.7c) admits a unique weak solution $y \in \mathcal{B}_{T^{*}}$ which satisfies (3.8) with $T=T^{*}$.

Proof. For $\theta \in(0, T]$, we define the operator $\Psi_{f, y_{0}}^{\theta}: \mathcal{B}_{\theta} \mapsto \mathcal{B}_{\theta}$ as

$$
\Psi_{f, y_{0}}(z)=\mathcal{L}\left(f-z \partial_{x} z, y_{0}\right)
$$

which is the weak solution of (3.1a)-(3.1c) with $T=\theta$ for the data $\left(f-z \partial_{x} z, y_{0}\right)$. Estimate (3.5) and Lemma 3.6 imply

$$
\begin{aligned}
\left\|\Psi_{f, y_{0}}(y)\right\|_{\mathcal{B}_{\theta}} & \leq C_{1}\left(\left\|y_{0}\right\|_{L^{2}(\Omega)}+\|f\|_{L^{2}\left(I, H^{-1}(\Omega)\right)}+\left\|y \partial_{x} y\right\|_{L^{2}\left(I, H^{-1}(\Omega)\right)}\right) \\
& \leq C_{1}\left(\left\|y_{0}\right\|_{L^{2}(\Omega)}+\|f\|_{L^{2}\left(I, H^{-1}(\Omega)\right)}\right)+C_{2} \theta^{1 / 4}\|y\|_{\mathcal{B}_{\theta}}^{2}
\end{aligned}
$$

Copyright (c) by SIAM. Unauthorized reproduction of this article is prohibited. 
and

$$
\left\|\Psi_{f, y_{0}}(y)-\Psi_{f, y_{0}}(z)\right\|_{\mathcal{B}_{\theta}} \leq C_{2} \theta^{1 / 4}\|y+z\|_{\mathcal{B}_{\theta}}\|y-z\|_{\mathcal{B}_{\theta}} .
$$

We choose a $\theta^{*}>0$ such that

$$
\left\{\begin{array}{l}
r=3 C_{1}\left(\left\|y_{0}\right\|_{L^{2}(\Omega)}+\|f\|_{L^{2}\left(0, T, H^{-1}(\Omega)\right)}\right), \\
C_{2} \theta^{* 1 / 4} r \leq \frac{1}{3}
\end{array}\right.
$$

holds and consider $B=\left\{y \in \mathcal{B}_{\theta^{*}}:\|y\|_{\mathcal{B}_{\theta^{*}}} \leq r\right\}$. Therefore, we have

$$
\left\|\Psi_{f, y_{0}}(y)\right\|_{\mathcal{B}_{\theta^{*}}} \leq \frac{1}{3} r+\frac{1}{3 r} r^{2}=\frac{2}{3} r
$$

and get

$$
\Psi_{f, y_{0}}(B) \subset B .
$$

Moreover we have

$$
\left\|\Psi_{f, y_{0}}(y)-\Psi_{f, y_{0}}(z)\right\|_{\mathcal{B}_{\theta^{*}}} \leq \frac{1}{3 r} 2 r\|y-z\|_{\mathcal{B}_{\theta^{*}}} \leq \frac{2}{3}\|y-z\|_{\mathcal{B}_{\theta^{*}}}
$$

for all $(y, z) \in B$. As a consequence, we can apply the Banach fixed point theorem which implies the existence of a unique fix point $y$ of $\Psi_{f, y_{0}}(\cdot)$.

According to the proof of Proposition 3.7, an upper bound for $\theta^{*}$ is given by

$$
\theta^{*} \leq \frac{C(T, L)}{\left(\left\|y_{0}\right\|_{L^{2}(\Omega)}+\|f\|_{L^{2}\left(I, H^{-1}(\Omega)\right)}\right)} .
$$

The bigger $\|f\|_{L^{2}\left(I, H^{-1}(\Omega)\right)}$ is, the shorter we can ensure the existence of the solution.

3.3. Time-global well-posedness of the KdVB equation with $\gamma=0$ and small data. In this section we establish the time-global wellposedness of the KdVB equation in the nonviscous case $\gamma=0$ and with small data. The inequality (3.13b) can be also satisfied for a fixed $T$ and small data, i.e., if

$$
\|f\|_{L^{2}\left(I, H^{-1}(\Omega)\right)}+\left\|y_{0}\right\|_{L^{2}(\Omega)} \leq \frac{C(T, L)}{T^{1 / 4}}
$$

is satisfied. In particular, we have the following result.

Corollary 3.8. Let $\gamma=0$. Then there exists a constant $C_{\max }(T, L)>0$ such that for any $\left(f, y_{0}\right) \in L^{2}\left(I, H^{-1}(\Omega)\right) \times L^{2}(\Omega)$ with

$$
\|f\|_{L^{2}\left(I, H^{-1}(\Omega)\right)}+\left\|y_{0}\right\|_{L^{2}(\Omega)} \leq C_{\max }
$$

there exists a unique solution $y \in \mathcal{B}$ of (3.7a)-(3.7c) which satisfies

$$
\|y\|_{\mathcal{B}} \leq c\left(\left\|y_{0}\right\|_{L^{2}(\Omega)}+\|f\|_{L^{2}\left(I, H^{-1}(\Omega)\right)}\right)
$$

for some $c(T, L)>0$ independent of $y$ and the data. Moreover there holds

$$
\left\|\partial_{t} y\right\|_{L^{2}\left(I, \mathcal{V}^{*}\right)} \leq \tilde{c}\left(L, T, y_{0}, f\right)
$$

for some constant $\tilde{c}>0$ only depending on $L, T, y_{0}, f$.

Copyright (C) by SIAM. Unauthorized reproduction of this article is prohibited. 
Proof. Existence, uniqueness, and (3.16) are proved using similar steps as in the proof of Proposition 3.7. In particular, the inequality (3.13b) is satisfied for our choice of data $\left(u, y_{0}\right)$. Now we proof the estimate (3.17) using (3.5), (3.16), and Lemma 3.6. In particular, we have

$$
\begin{aligned}
\left\|\partial_{t} y\right\|_{L^{2}\left(I, \mathcal{V}^{*}\right)} & \leq c\left(\left\|y_{0}\right\|_{L^{2}(\Omega)}+\left\|y \partial_{x} y\right\|_{L^{2}\left(I, H^{-1}(\Omega)\right)}+\|f\|_{L^{2}\left(I, H^{-1}(\Omega)\right)}\right) \\
& \leq c\left(\left\|y_{0}\right\|_{L^{2}(\Omega)}+\|y\|_{\mathcal{B}}^{2}+\|f\|_{L^{2}\left(I, H^{-1}(\Omega)\right)}\right) \\
& \leq \tilde{c}\left(T, L, y_{0}, f\right) .
\end{aligned}
$$

3.3.1. Time-global well-posedness of the KdVB equation with $\gamma>0$ and general data. In this section we prove time-global wellposedness of the KdVB equation in the viscous case and with general data. Proposition 3.7 guarantees local well-posedness in time. Therefore, global well-posedness will follow from a priori estimates for the nonlinear problem. We will show that such estimates exist in the viscous case $\gamma>0$.

Theorem 3.9. Let $\gamma>0$. For any $\left(f, y_{0}\right) \in L^{2}\left(I, H^{-1}(\Omega)\right) \times L^{2}(\Omega)$ let $y \in \mathcal{B}_{\theta}$, $\theta \in(0, T]$, be a time-local solution of $(3.7 \mathrm{a})-(3.7 \mathrm{c})$, and then $y$ satisfies

$$
\|y\|_{\mathcal{B}_{\theta}} \leq c\left(\frac{\sqrt{\gamma}+1}{\sqrt{\gamma}}\right)\left(\left\|y_{0}\right\|_{L^{2}(\Omega)}+\frac{1}{\sqrt{\gamma}}\|f\|_{L^{2}\left(I, H^{-1}(\Omega)\right)}\right)
$$

for some $c>0$ independent of $y$ and the data.

Proof. For a classical solution $\tilde{y}$ of the linear equation with the right-hand side $g$ we have

$$
\frac{1}{2} \frac{d}{d t}\|\tilde{y}(t)\|_{L^{2}(\Omega)}^{2}+\frac{1}{2}\left|\partial_{x} \tilde{y}(t, 0)\right|^{2}+\gamma\|\tilde{y}(t)\|_{H_{0}^{1}(\Omega)}^{2}=\langle g(t), \tilde{y}(t)\rangle_{H^{-1}(\Omega), H_{0}^{1}(\Omega)} ;
$$

see (A.1). Then we omit the positive term $\left|\partial_{x} \tilde{y}(t, 0)\right|^{2}$, integrate over $(0, t), 0<t \leq \theta$, and get

$$
\frac{1}{2}\|\tilde{y}(t)\|_{L^{2}(\Omega)}^{2}+\gamma \int_{0}^{t}\|\tilde{y}\|_{H_{0}^{1}(\Omega)}^{2} \mathrm{~d} s \leq \frac{1}{2}\left\|y_{0}\right\|_{L^{2}(\Omega)}+\int_{0}^{t}\langle g, \tilde{y}\rangle_{H^{-1}(\Omega), H_{0}^{1}(\Omega)} \mathrm{d} s .
$$

This inequality also holds for a weak solution of the linear equation using a density argument. Since $y \in \mathcal{B}_{\theta}$ is a fixed point of the nonlinear equation we have

$$
\frac{1}{2}\|y(t)\|_{L^{2}(\Omega)}^{2}+\gamma \int_{0}^{t}\|y\|_{H_{0}^{1}(\Omega)}^{2} \mathrm{~d} s \leq \frac{1}{2}\left\|y_{0}\right\|_{L^{2}(\Omega)}^{2}+\int_{0}^{t}\left\langle f-y \partial_{x} y, y\right\rangle_{H^{-1}(\Omega), H_{0}^{1}(\Omega)} \mathrm{d} s .
$$

Due to the fact that

$$
\int_{0}^{t}\left\langle y \partial_{x} y, y\right\rangle_{H^{-1}(\Omega), H_{0}^{1}(\Omega)} \mathrm{d} s=-\frac{1}{6} \int_{0}^{t} \int_{\Omega} \partial_{x}\left(y^{3}\right) \mathrm{d} x \mathrm{~d} s=0
$$

for any $t \in[0, \theta]$ we get

$$
\frac{1}{2}\|y(t)\|_{L^{2}(\Omega)}^{2}+\gamma \int_{0}^{t}\|y\|_{H_{0}^{1}(\Omega)}^{2} \mathrm{~d} s \leq \frac{1}{2}\left\|y_{0}\right\|_{L^{2}(\Omega)}^{2}+\int_{0}^{t} \frac{1}{2 \gamma}\|f\|_{H^{-1}(\Omega)}^{2}+\frac{\gamma}{2}\|y\|_{H_{0}^{1}(\Omega)}^{2} \mathrm{~d} s .
$$

Then subtracting the term $\frac{\gamma}{2} \int_{0}^{t}\|y\|_{H_{0}^{1}(\Omega)}^{2} \mathrm{~d} s$ yields

$$
\frac{1}{2}\|y(t)\|_{L^{2}(\Omega)}^{2}+\frac{\gamma}{2} \int_{0}^{t}\|y\|_{H_{0}^{1}(\Omega)}^{2} \mathrm{~d} s \leq \frac{1}{2}\left\|y_{0}\right\|_{L^{2}(\Omega)}^{2}+\frac{2}{\gamma}\|f\|_{L^{2}\left(I, H^{-1}(\Omega)\right)}^{2} .
$$

Copyright (c) by SIAM. Unauthorized reproduction of this article is prohibited. 
Omitting the term $\frac{\gamma}{2} \int_{0}^{t}\|y\|_{H_{0}^{1}(\Omega)}^{2} \mathrm{~d} s$ yields

$$
\|y(t)\|_{L^{2}(\Omega)}^{2} \leq\left\|y_{0}\right\|_{L^{2}(\Omega)}^{2}+\frac{1}{\gamma}\|f\|_{L^{2}\left(I, H^{-1}(\Omega)\right)}^{2} .
$$

Now we set $t=\theta$ in (3.19) and omit the term $\|y(\theta)\|_{L^{2}(\Omega)}^{2}$ and get

$$
\|y\|_{L^{2}\left((0, \theta), H_{0}^{1}(\Omega)\right)}^{2} \leq \frac{1}{\gamma}\left\|y_{0}\right\|_{L^{2}(\Omega)}^{2}+\frac{1}{\gamma^{2}}\|f\|_{L^{2}\left(I, H^{-1}(\Omega)\right)}^{2} .
$$

Adding (3.20) and (3.21) gives the global estimate (3.18).

Remark 3.10. The constant in the estimate (3.18) explodes for $\gamma \rightarrow 0$.

Corollary 3.11. Let $\gamma>0$ and $\left(f, y_{0}\right) \in L^{2}\left(I, H^{-1}(\Omega)\right) \times L^{2}(\Omega)$. Then there exists a unique solution $y \in \mathcal{B} \cap L^{2}\left(I, \mathcal{V}^{*}\right)$ of $(3.7 \mathrm{a})-(3.7 \mathrm{c})$ which satisfies

$$
\|y\|_{\mathcal{B}} \leq c(\gamma)\left(\left\|y_{0}\right\|_{L^{2}(\Omega)}+\|f\|_{L^{2}\left(I, H^{-1}(\Omega)\right)}\right)
$$

for some $c>0$ independent of $y$ and the data. Moreover there holds

$$
\left\|\partial_{t} y\right\|_{L^{2}\left(I, \mathcal{V}^{*}\right)} \leq C\left(T, L, y_{0}, f, \gamma\right)
$$

for some $C>0$ only depending on $L, T, y_{0}, f$, and $\gamma$.

Proof. First, we discuss uniqueness. Let $y_{1}$ and $y_{2}$ two solutions of (3.7a) $-(3.7 \mathrm{c})$. We define $\delta y=y_{1}-y_{2}$. Then $\delta y$ solves (A.6a)-(A.6c) with $y=y_{1}+y_{2}$. Then Proposition A.29 implies $y_{1}=y_{2}$. According to Proposition $3.7(3.7 \mathrm{a})-(3.7 \mathrm{c})$ has a unique time-local solution $y \in \mathcal{B}_{T^{*}}$. Thus we can repeat the procedure of Proposition 3.7 starting from $y\left(T^{*}\right)$ until a maximal time of existence $0<t_{\max } \leq T$ is reached. If $t_{\max }<T$ it follows from [42, Chapter 6, Theorem 2.2] that $\lim _{t \uparrow t_{\max }}\|y(t)\|_{L^{2}(\Omega)}=\infty$, which contradicts (3.18). The estimate (3.23) is proved using (3.18) and similar arguments as in the proof of Corollary 3.8.

4. Well-posedness of the optimization problem. Existence of an optimum. We start by defining the admissible set of controls

$$
U_{a d}= \begin{cases}\left\{u \in \mathcal{M}\left(\Omega_{c}, L^{2}(I)\right):\|u\|_{\mathcal{M}\left(\Omega_{c}, L^{2}(I)\right)} \leq c_{a d}\right\} & \text { if } \gamma=0, \\ \mathcal{M}\left(\Omega_{c}, L^{2}(I)\right) & \text { otherwise, }\end{cases}
$$

where $c_{a d}<c_{\max }:=\left(C_{\max }-\left\|y_{0}\right\|_{L^{2}(\Omega)}\right) / \hat{c}$, where $\hat{c}$ is the embedding constant of $\mathcal{M}\left(\Omega_{c}, L^{2}(I)\right) \hookrightarrow L^{2}\left(I, H^{-1}(\Omega)\right)$. The constant $C_{\max }$ comes from (3.15) and we assume that

$$
\left\|y_{0}\right\|_{L^{2}(\Omega)}<C_{\max } .
$$

Moreover we introduce the nonlinear control-to-state operator

$$
S: U_{a d} \rightarrow \mathcal{B} \cap H^{1}\left(I, \mathcal{V}^{*}\right), \quad u \mapsto y,
$$

where $y$ is a weak solution of (1.2a)-(1.2c) for a given $y_{0} \in L^{2}(\Omega)$ which satisfies (4.1) in the case $\gamma=0$. According to subsection 3.3 the mapping $S$ is well-defined. In [16] the authors used a similar strategy for the definition of the control-to-state mapping. The control-to-observation operator is denoted by

$$
S_{o b s}: U_{a d} \rightarrow L^{2}\left(I \times \Omega_{o}\right) \times L^{2}\left(\Omega_{o}\right), \quad u \mapsto\left(\chi_{\Omega_{o}} y, \chi_{\Omega_{o}} y(T)\right) .
$$


Thus we can rewrite problem $(\mathcal{P})$ in its reduced form given by

$$
\min _{u \in U_{a d}} \frac{1}{2}\left\|S_{o b s}(u)-z\right\|_{L^{2}\left(I \times \Omega_{o}\right) \times L^{2}\left(\Omega_{o}\right)}^{2}+\alpha\|u\|_{\mathcal{M}\left(\Omega_{c}, L^{2}(I)\right)}
$$

with $z \in L^{2}\left(I \times \Omega_{o}\right) \times L^{2}\left(\Omega_{o}\right)$.

Proposition 4.12. There exists a global solution $(\bar{y}, \bar{u}) \in \mathcal{B} \times U_{\text {ad }}$ to the optimal control problem $(\mathcal{P})$.

Proof. Case $\gamma>0$. The cost function $J$ is a positive function. Thus its infimum $\bar{J}$ exists and there exists a minimizing sequence $\left(u_{n}, y_{n}\right) \in U_{a d} \times \mathcal{B}$ such that $J\left(u_{n}, y_{n}\right) \rightarrow$ $\bar{J}$ as $n \rightarrow \infty$. Furthermore there exists an $\varepsilon>0$ such that

$$
\varepsilon+J(S(0), 0) \geq \alpha\left\|u_{n}\right\|_{\mathcal{M}\left(\Omega_{c}, L^{2}(I)\right)}
$$

for $n$ large enough. Therefore $u_{n}$ is bounded, which implies the existence of an element $\bar{u} \in \mathcal{M}\left(\Omega_{c}, L^{2}(I)\right)$ and a subsequence $u_{n_{k}}$ converging in the weak-* topology of $\mathcal{M}\left(\Omega_{c}, L^{2}(I)\right)$ toward $\bar{u}$; cf. [9, Corollary 3.30]. For each $u_{n_{k}}$, we define $y_{n_{k}}=$ $S u_{n_{k}} \in \mathcal{B} \cap H^{1}\left(I, \mathcal{V}^{*}\right)$. Thanks to the global estimate (3.18), there exists a exists a $\bar{y} \in L^{2}\left(I, H_{0}^{1}(\Omega)\right) \cap L^{\infty}\left(I, L^{2}(\Omega)\right) \cap H^{1}\left(I, \mathcal{V}^{*}\right)$ such that

$$
y_{n_{k}} \rightarrow^{*} \bar{y} \quad \text { in } \quad L^{2}\left(I, H_{0}^{1}(\Omega)\right) \cap L^{\infty}\left(I, L^{2}(\Omega)\right) \cap H^{1}\left(I, \mathcal{V}^{*}\right)
$$

and a $\hat{y} \in L^{2}(\Omega)$ such that $y_{n_{k}}(T) \rightarrow \hat{y}$ in $L^{2}(\Omega)$ up to a subsequence. Moreover the Aubin-Lions lemma (cf. [49, Chapter 3, Proposition 1.3]) implies

$$
y_{n_{k}} \rightarrow \bar{y} \quad \text { in } L^{2}\left(I, \mathcal{C}_{0}(\Omega)\right) .
$$

It remains to show that the limit $\bar{y}$ is indeed a weak solution of (1.2a)-(1.2c) for the control $\bar{u}$. Due to the weak-to-weak continuity of time-point evaluation operator $y \mapsto y(T)$ from $H^{1}\left(I, \mathcal{V}^{*}\right)$ to $\mathcal{V}^{*}$ there holds $\hat{y}=y(T)$. The convergence of the linear terms in (3.8) is obvious. The nonlinear term converges due to the strong convergence of $y_{n_{k}}$, since

$$
\begin{aligned}
\int_{0}^{T}\left\langle y_{n_{k}} \partial_{x} y_{n_{k}}-\bar{y} \partial_{x} \bar{y}, p\right\rangle_{H^{-1}(\Omega), H_{0}^{1}(\Omega)} \mathrm{d} t=-\int_{0}^{T}\left(y_{n_{k}}^{2}-\bar{y}^{2}, \partial_{x} p\right)_{L^{2}(\Omega)} \mathrm{d} t \\
\quad \leq\left\|y_{n_{k}}-\bar{y}\right\|_{L^{2}\left(I, \mathcal{C}_{0}(\Omega)\right)}\left\|y_{n_{k}}+\bar{y}\right\|_{\mathcal{C}\left(\bar{I}, L^{2}(\Omega)\right)}\left\|\partial_{x} p\right\|_{L^{2}(I \times \Omega)} .
\end{aligned}
$$

Therefore $\bar{y}$ is a weak solution of $(1.2 \mathrm{a})-(1.2 \mathrm{c})$ for the control $\bar{u}$ and it holds that $\bar{y} \in \mathcal{B} \cap H^{1}\left(I, \mathcal{V}^{*}\right)$. Moreover there holds

$$
S_{o b s}\left(u_{n_{k}}\right) \rightarrow S_{o b s}(\bar{u}) \quad \text { in } L^{2}\left(I \times \Omega_{o}\right) \times L^{2}\left(\Omega_{o}\right) .
$$

The tracking functional is weak lower semicontinuous in $L^{2}\left(I \times \Omega_{o}\right) \times L^{2}\left(\Omega_{o}\right)$ and the control cost term is weak-* lower semicontinuous in $\mathcal{M}\left(\Omega_{c}, L^{2}(I)\right)$; therefore $(\bar{y}, \bar{u})$ is a solution of $(\mathcal{P})$.

Case $\gamma=0$. In this case we rely on the estimate (3.16) and use that the set $U_{a d}$ is weak-* closed in $\mathcal{M}\left(\Omega_{c}, L^{2}(I)\right)$.

Remark 4.13. We know for a minimizing sequence $\left(u_{n}, y_{n}\right) \in \mathcal{M}\left(\Omega_{c}, L^{2}(I)\right) \times \mathcal{B}$ that there exists an $\varepsilon>0$ and an $N \in \mathbb{N}$ such that for any $n>N$

$$
\left\|u_{n}\right\|_{\mathcal{M}\left(\Omega_{c}, L^{2}(I)\right)} \leq \frac{c}{\alpha}(J(S(0), 0)+\varepsilon) .
$$

Therefore, we can always find a regularization parameter $\alpha>0$ such that the condition (3.15) is satisfied. 
5. First order optimality conditions. First we discuss the differentiability of the control-to-state operator $S$ defined in (4.2). In order to do that, we introduce the open set

$$
\tilde{U}_{a d}= \begin{cases}\left\{u \in \mathcal{M}\left(\Omega_{c}, L^{2}(I)\right):\|u\|_{\mathcal{M}\left(\Omega_{c}, L^{2}(I)\right)}<\hat{c}_{a d}\right\} & \text { if } \gamma=0, \\ \mathcal{M}\left(\Omega_{c}, L^{2}(I)\right) & \text { otherwise }\end{cases}
$$

with $c_{a d}<\hat{c}_{a d}<c_{\max }$. The following lemma concerning the local Lipschitz continuity of $S$ is needed for the proof of its Fréchet differentiability.

Lemma 5.14. The control-to-state operator $S: \tilde{U}_{a d} \rightarrow \mathcal{B}$ is locally Lipschitz continuous, i.e., for every $u \in \tilde{U}_{\text {ad }}$ there exists a neighborhood $V \subset \tilde{U}_{\text {ad }}$ and a constant $C(T, L, u, \tilde{u})>0$ such that

$$
\|S(u)-S(\tilde{u})\|_{\mathcal{B}} \leq C(T, L, u, \tilde{u})\|u-\tilde{u}\|_{\mathcal{M}\left(\Omega_{c}, L^{2}(I)\right)} \quad \forall \tilde{u} \in V .
$$

Proof. We define $y=S(u) \in \mathcal{B}$ and $\tilde{y}=S(\tilde{u}) \in \mathcal{B}$ for $\tilde{u} \in V$. Therefore, the difference $w=\tilde{y}-y$ satisfies the equation

$$
\left\{\begin{array}{l}
\partial_{t} w+\partial_{x} w+\partial_{x x x} w-\gamma \partial_{x x} w+\frac{1}{2} \partial_{x}((y+\tilde{y}) w)=\tilde{u}-u \text { in } I \times \Omega \\
w \cdot, 0)=w(\cdot, L)=\partial_{x} w(\cdot, L)=0 \text { in } I \\
w(0, x)=0 \text { in } \Omega
\end{array}\right.
$$

in the weak sense of (3.8). According to Appendix A.3 $w \in \mathcal{B}$ satisfies the a priori estimates

$$
\|w\|_{\mathcal{B}} \leq C(T, L, u, \tilde{u})\|u-\tilde{u}\|_{\mathcal{M}\left(\Omega_{c}, L^{2}(I)\right)} .
$$

Thus we can conclude that the solution operator $S$ is locally Lipschitz continuous.

Proposition 5.15. The control to state operator $S: \tilde{U}_{a d} \rightarrow \mathcal{B}$ is continuously Fréchet-differentiable. Its derivative

$$
S^{\prime}(u): \mathcal{M}\left(\Omega_{c}, L^{2}(I)\right) \rightarrow \mathcal{B}, \delta u \mapsto \delta y
$$

at $u \in U_{a d}$ is given by the solution operator of the linear tangent equation

$$
\left\{\begin{array}{l}
\partial_{t} \delta y+\partial_{x} \delta y-\gamma \partial_{x x} \delta y+\partial_{x x x} \delta y+\partial_{x}(y \delta y)=\delta u \text { in } I \times \Omega \\
\delta y(\cdot, 0)=\delta y(\cdot, L)=\partial_{x} \delta y(\cdot, L)=0 \text { on } I \\
\delta y(0, \cdot)=0 \text { on } \Omega
\end{array}\right.
$$

Proof. First we mention that $S$ is well-defined on $\tilde{U}_{a d}$ and that the nonlinearity $F: \mathcal{B} \rightarrow L^{2}\left(I, H^{-1}(\Omega)\right), y \mapsto y \partial_{x} y$ is Fréchet differentiable since there holds

$$
\left\|F(y+\delta y)-F(y)-F^{\prime}(y) \delta y\right\|_{L^{2}\left(I, H^{-1}(\Omega)\right)} \leq \frac{1}{2}\|\delta y\|_{L^{4}(I \times \Omega)}^{2} \leq c\|\delta y\|_{\mathcal{B}}^{2}
$$

with $F^{\prime}(y) \delta y=\partial_{x}(y \delta y)$ for any $\delta y \in \mathcal{B} \hookrightarrow L^{4}(I \times \Omega)$. Then we differentiate the fixed point equation $y=\mathcal{L}\left(u-y \partial_{x} y, y_{0}\right)$ with respect to $(y, u)$ in direction $(\delta y, \delta u) \in$ $\mathcal{B} \times \mathcal{M}\left(\Omega_{c}, L^{2}(I)\right)$ and get

$$
\delta y=\mathcal{L}^{\prime}\left(\delta u-\partial_{x}(y \delta y)\right)
$$

Copyright $@$ by SIAM. Unauthorized reproduction of this article is prohibited. 
where $\mathcal{L}^{\prime}(\cdot)=\mathcal{L}(\cdot, 0)$. In the Appendix A.3 it is shown that (5.2) has a unique solution $\delta y \in \mathcal{B}$. Actually $\delta y$ is the weak solution of (5.1a)-(5.1c) in the sense of (3.8). Next we show that $S^{\prime}(u) \delta u:=\delta y$ is the Fréchet derivative of $S$. This will result from the study of

$$
\frac{1}{\|\delta u\|_{\mathcal{M}\left(\Omega_{c}, L^{2}(I)\right)}}\left\|S(u+\delta u)-S(u)-S^{\prime}(u) \delta u\right\|_{\mathcal{B}}=\frac{1}{\|\delta u\|_{\mathcal{M}\left(\Omega_{c}, L^{2}(I)\right)}}\|\tilde{y}-y-\delta y\|_{\mathcal{B}}
$$

Calling $w=\tilde{y}-y-\delta y \in \mathcal{B}$ the function $w$ then satisfies

$$
\left\{\begin{array}{l}
\partial_{t} w+\partial_{x} w+\partial_{x x x} w-\gamma \partial_{x x} w+\tilde{y} \partial_{x} \tilde{y}-y \partial_{x} y-\partial_{x}(y \delta y)=0 \text { in } I \times \Omega \\
w(\cdot, 0)=w(\cdot, L)=\partial_{x} w(\cdot, L)=0 \text { in } I \\
w(0, \cdot)=0 \text { in } \Omega
\end{array}\right.
$$

in the weak sense of (3.8). After rearranging the terms we end up with

$$
\left\{\begin{array}{l}
\partial_{t} w+\partial_{x} w+\partial_{x x x} w-\gamma \partial_{x x} w+\partial_{x}(y w)=-(\tilde{y}-y) \partial_{x}(\tilde{y}-y) \text { in } I \times \Omega \\
w(\cdot, 0)=w(\cdot, L)=\partial_{x} w(\cdot, L)=0 \text { in } I \\
w(0, \cdot)=0 \text { in } \Omega
\end{array}\right.
$$

According to Appendix A.3 and Lemma 3.6 it holds that

$$
\|w\|_{\mathcal{B}} \leq C(T, L, u)\left\|(\tilde{y}-y) \partial_{x}(\tilde{y}-y)\right\|_{L^{2}\left(I, H^{-1}(\Omega)\right)} \leq C(T, L, u) T^{1 / 4}\|\tilde{y}-y\|_{\mathcal{B}}^{2} .
$$

Therefore the conclusion follows from local Lipschitz continuity of $S$; see Lemma 5.14. Hence we provide the following lemma, which concludes the proof.

Therefore the control-to-observation operator $S_{o b s}$ is also Fréchet differentiable and its derivative is given by

$$
S_{o b s}^{\prime}(u): \delta u \mapsto\left(\chi_{\Omega_{o}} \delta y, \chi_{\Omega_{o}} \delta y(T)\right),
$$

where $\delta y \in \mathcal{B}$ is the weak solution of (5.1). Next we introduce the adjoint control to observation operator $S^{\prime \star}$.

Proposition 5.16. Let $u \in U_{a d}$. There exists a bounded linear operator

$$
S_{o b s}^{\prime \star}(u): L^{2}\left(I \times \Omega_{o}\right) \times L^{2}\left(\Omega_{o}\right) \rightarrow \mathcal{C}\left(\Omega_{c}, L^{2}(I)\right),\left(\phi, p_{T}\right) \mapsto p
$$

which fulfills

$$
\begin{aligned}
& \left(S_{o b s}^{\prime}(u) \delta u, \phi\right)_{L^{2}\left(I \times \Omega_{o}\right)}+\left(\left(S_{o b s}^{\prime}(u) \delta u\right)(T), p_{T}\right)_{L^{2}\left(\Omega_{o}\right)} \\
& =\left\langle\delta u, S_{o b s}^{\prime \star}(u)\left(\phi, p_{T}\right)\right\rangle_{\mathcal{M}\left(\Omega_{c}, L^{2}(I)\right), \mathcal{C}\left(\Omega_{c}, L^{2}(I)\right)} \\
& \quad \forall \delta u \in \mathcal{M}\left(\Omega_{c}, L^{2}(I)\right),\left(\phi, p_{T}\right) \in L^{2}\left(I \times \Omega_{o}\right) \times L^{2}\left(\Omega_{o}\right) .
\end{aligned}
$$

Moreover it is the solution operator of

$$
\left\{\begin{array}{l}
-\partial_{t} p-\partial_{x} p-\gamma \partial_{x x} p-\partial_{x x x} p-y \partial_{x} p=\phi \text { in } I \times \Omega \\
p(\cdot, 0)=p(\cdot, L)=\partial_{x} p(\cdot, 0)=0 \text { in } I \\
p(T, \cdot)=p_{T} \text { in } \Omega
\end{array}\right.
$$

with $y=S(u)$ for $\left(\phi, p_{T}\right) \in L^{2}\left(I \times \Omega_{o}\right) \times L^{2}\left(\Omega_{o}\right)$. 
Proof. First we mention that $y \partial_{x} p \in L^{1}\left(I, L^{2}(\Omega)\right)$ holds for $y \in \mathcal{B}$ and $p \in \mathcal{B}$; cf. Lemma A.31. We use the weak formulation of the tangent equation and get

$$
\begin{aligned}
\left(S_{o b s}^{\prime}(u) \delta u, \phi\right)_{L^{2}\left(I \times \Omega_{o}\right)}+\left(\left(S_{o b s}^{\prime}(u) \delta u\right)(T), p_{T}\right)_{L^{2}\left(\Omega_{o}\right)} & \\
=\left(\delta y, \chi_{\Omega_{o}}^{*} \phi\right)_{L^{2}(I \times \Omega)}+\left(\delta y(T), \chi_{\Omega_{o}}^{*} p_{T}\right)_{L^{2}(\Omega)} & -\int_{0}^{T}\left(\delta y, y \partial_{x} p\right)_{L^{2}(\Omega)} \mathrm{d} t \\
& =\langle\delta u, p\rangle_{\mathcal{M}\left(\Omega_{c}, L^{2}(I)\right), \mathcal{C}\left(\Omega_{c}, L^{2}(I)\right)}
\end{aligned}
$$

for $\delta u \in \mathcal{M}\left(\Omega_{c}, L^{2}(I)\right)$ and $y=S(u) \in \mathcal{B}$, where $\chi_{\Omega_{o}}^{*}$ is the extension operator to $\Omega$. By comparing with (5.3), we set $S_{o b s}^{\prime \star}(u)\left(\phi, p_{T}\right):=\chi_{\Omega_{c}} p$, where $p$ solves the fixed point equation

$$
p(t)=W^{*}(T-t) p_{T}+\int_{t}^{T} W^{*}(s-t)\left(\phi(s)-y(s) \partial_{x} p(s)\right) \mathrm{d} s, \quad t \in I .
$$

In Appendix A.4 we show that the fixed point equation (5.6) has a unique solution $p \in \mathcal{B} \hookrightarrow \mathcal{C}\left(\Omega_{c}, L^{2}(I)\right)$ which depends continuously on $\left(\phi, p_{T}\right)$.

Next we derive first order optimality conditions using tools from convex analysis.

Proposition 5.17. Let $(\bar{y}, \bar{u}) \in \mathcal{B} \times U_{a d}$ be a local solution of $(\mathcal{P})$. Then $\bar{u}$ satisfies the following variational variational inequality

$\left\langle-\chi_{\Omega_{c}} \bar{p}, u-\bar{u}\right\rangle_{\mathcal{C}\left(\Omega_{c}, L^{2}(I)\right), \mathcal{M}\left(\Omega_{c}, L^{2}(I)\right)}+\|\bar{u}\|_{\mathcal{M}\left(\Omega_{c}, L^{2}(I)\right)} \leq\|u\|_{\mathcal{M}\left(\Omega_{c}, L^{2}(I)\right)} \quad \forall u \in U_{a d}$,

where $\bar{p}$ is the solution of the adjoint state equation

$$
\left\{\begin{array}{l}
-\partial_{t} \bar{p}-\partial_{x} \bar{p}-\gamma \partial_{x x} \bar{p}-\partial_{x x x} \bar{p}-\bar{y} \partial_{x} \bar{p}=\chi_{\Omega_{o}} \bar{y}-z_{1} \text { in } \Omega, \\
\bar{p}(\cdot, 0)=\bar{p}(\cdot, L)=\partial_{x} \bar{p}(\cdot, 0)=0 \text { in } I, \\
\bar{p}(T, \cdot)=\chi_{\Omega_{o}} y(T)-z_{2} \text { in } \Omega .
\end{array}\right.
$$

Proof. We define

$$
F\left(u_{1}, u_{2}\right)=\frac{1}{2}\left(\left\|y_{1}-z_{1}\right\|_{L^{2}\left(I \times \Omega_{o}\right)}^{2}+\left\|y_{2}-z_{2}\right\|_{L^{2}\left(\Omega_{o}\right)}^{2}\right)
$$

for $\left(y_{1}, y_{2}\right) \in L^{2}\left(I \times \Omega_{o}\right) \times L^{2}\left(\Omega_{o}\right)$ and $\psi(u)=\alpha\|u\|_{\mathcal{M}\left(\Omega_{c}, L^{2}(I)\right)}$. Since $F$ and $S_{\text {obs }}$ are Fréchet differentiable the directional derivative of $F \circ S_{o b s}$ at $\bar{u}$ has the form

$D\left(F \circ S_{o b s}, \bar{u}, \delta u\right)=\left\langle S_{o b s}^{\prime \star}(\bar{u})\left(S_{o b s}(\bar{u})-z\right), \delta u\right\rangle_{\mathcal{C}\left(\Omega_{c}, L^{2}(I)\right), \mathcal{M}\left(\Omega_{c}, L^{2}(I)\right)}, \quad \delta u \in \mathcal{C}\left(\Omega_{c}, L^{2}(I)\right)$.

Then we set $\chi_{\Omega_{c}} \bar{p}:=S_{o b s}^{\prime \star}(\bar{u})\left(S_{o b s}(\bar{u})-z\right)$. An element $\bar{u} \in U_{a d}$ is local optimal if and only if there exists a $\delta>0$ such that

$$
F \circ S_{o b s}(\bar{u})+\psi(\bar{u}) \leq F \circ S_{o b s}(u)+\psi(u) \quad \forall u \in U_{a d} \cap B_{\delta}(\bar{u}) .
$$

In particular, there holds

$$
F \circ S_{o b s}(\bar{u})+\psi(\bar{u}) \leq F \circ S_{o b s}(\bar{u}+\varepsilon(u-\bar{u}))+\psi(\bar{u}+\varepsilon(u-\bar{u})) \quad \forall u \in U_{a d}
$$

for some $0<\varepsilon$ small enough. Using the convexity of $\psi$ we get

$$
\frac{F \circ S_{o b s}(\bar{u})-F \circ S_{o b s}(\bar{u}+\varepsilon(u-\bar{u}))}{\varepsilon}+\psi(\bar{u}) \leq \psi(u),
$$

which implies

$$
\langle-\bar{p}, u-\bar{u}\rangle_{\mathcal{C}\left(\Omega_{c}, L^{2}(I)\right), \mathcal{M}\left(\Omega_{c}, L^{2}(I)\right)}+\psi(\bar{u}) \leq \psi(u) \quad \forall u \in U_{a d} .
$$

Copyright ( by SIAM. Unauthorized reproduction of this article is prohibited. 
The subgradient conditions can be equivalently reformulated in the following form.

Proposition 5.18. The subgradient condition (5.7) is equivalent to

$$
\alpha\|\bar{u}\|_{\mathcal{M}\left(\Omega_{c}, L^{2}(I)\right)}+\phi^{\star}\left(-\chi_{\Omega_{c}} \bar{p}\right)=\left\langle-\chi_{\Omega_{c}} \bar{p}, \bar{u}\right\rangle_{\mathcal{C}\left(\Omega_{c}, L^{2}(I)\right), \mathcal{M}\left(\Omega_{c}, L^{2}(I)\right)}, \quad \bar{u} \in U_{a d},
$$

with

$$
\phi^{\star}(p)=\sup _{u \in U_{a d}}\left[\langle u, p\rangle_{\mathcal{C}\left(\Omega_{c}, L^{2}(I)\right), \mathcal{M}\left(\Omega_{c}, L^{2}(I)\right)}-\alpha\|u\|_{\mathcal{M}\left(\Omega_{c}, L^{2}(I)\right)}\right]
$$

for $p \in \mathcal{C}\left(\Omega_{c}, L^{2}(I)\right)$.

Proof. This is a well known characterization of the subdifferential of a convex function; cf. [23]. However the assertion can be easily derived from the definition of the of $\phi^{\star}$ and (5.7).

Next we characterize $\phi^{\star}$.

Lemma 5.19. The functional $\phi^{\star}(p): \mathcal{C}\left(\Omega_{c}, L^{2}(I)\right) \rightarrow \mathbb{R}$ has the form

$$
\phi^{\star}(p)= \begin{cases}c_{a d}\left(\|p\|_{\mathcal{C}\left(\Omega_{c}, L^{2}(I)\right)}-\alpha\right)^{+} & \gamma=0 \\ \mathcal{I}_{\mathcal{C}\left(\Omega_{c}, L^{2}(I)\right)}(p) & \gamma>0\end{cases}
$$

where $(\cdot)^{+}=\max (0, \cdot)$ and

$$
\mathcal{I}_{\mathcal{C}\left(\Omega_{c}, L^{2}(I)\right)}(p)= \begin{cases}0 & \|p\|_{\mathcal{C}\left(\Omega_{c}, L^{2}(I)\right)} \leq \alpha \\ \infty & \text { else }\end{cases}
$$

Proof. In the case $\gamma>0$ we set $b=\infty$ and $b=c_{a d}$ else. Then we have

$$
\begin{aligned}
\phi^{\star}(p) & =\sup _{u \in U_{a d}}\left[\langle u, p\rangle_{\mathcal{C}\left(\Omega_{c}, L^{2}(I)\right), \mathcal{M}\left(\Omega_{c}, L^{2}(I)\right)}-\alpha\|u\|_{\mathcal{M}\left(\Omega_{c}, L^{2}(I)\right)}\right] \\
& =\sup _{\lambda \in(0, b]\|u\|_{\mathcal{M}\left(\Omega_{c}, L^{2}(I)\right)}=\lambda}\left[\langle u, p\rangle_{\mathcal{C}\left(\Omega_{c}, L^{2}(I)\right), \mathcal{M}\left(\Omega_{c}, L^{2}(I)\right)}-\alpha \lambda\right] \\
& =\sup _{\lambda \in(0, b]} \lambda\left[\|p\|_{\mathcal{C}\left(\Omega_{c}, L^{2}(I)\right)}-\alpha\right],
\end{aligned}
$$

since

$$
\lambda\|p\|_{\mathcal{C}\left(\Omega_{c}, L^{2}(I)\right)}=\sup _{\|u\|_{\mathcal{M}\left(\Omega_{c}, L^{2}(I)\right)}=\lambda}\langle u, p\rangle_{\mathcal{C}\left(\Omega_{c}, L^{2}(I)\right), \mathcal{M}\left(\Omega_{c}, L^{2}(I)\right)} .
$$

Therefore, we have

$$
\phi^{\star}(p)= \begin{cases}0 & \|p\|_{\mathcal{C}\left(\Omega_{c}, L^{2}(I)\right)} \leq \alpha, \\ b\left(\|p\|_{\mathcal{C}\left(\Omega_{c}, L^{2}(I)\right)}-\alpha\right) & \text { else. }\end{cases}
$$

This implies the assertion.

Using Proposition 5.18 and Lemma 5.19 we can derive the following structural properties of the optimal control.

Proposition 5.20. Let $\bar{u} \in U_{\text {ad }}$ be a local solution of $(\mathcal{P})$. Moreover let $|\bar{u}| \in$ $\mathcal{M}(\Omega)$ be its total-variation measure and $\bar{u}^{\prime}$ its corresponding Radon-Nikodym-derivative. Furthermore let $\bar{p}$ be the corresponding optimal adjoint state. Then there holds the following:

Copyright $@$ by SIAM. Unauthorized reproduction of this article is prohibited. 
1. Nonviscous case $\gamma=0$ :

$$
\begin{aligned}
0 & =\left(\|\bar{u}\|_{\mathcal{M}\left(\Omega_{c}, L^{2}(I)\right)}-c_{a d}\right) \bar{\lambda} \\
\operatorname{supp}|\bar{u}| & \subseteq\left\{x \in \Omega_{c}:\|\bar{p}\|_{L^{2}(I)}=\alpha+\bar{\lambda}\right\}
\end{aligned}
$$

with $\bar{\lambda}=\left(\|\bar{p}\|_{\mathcal{C}\left(\Omega_{c}, L^{2}(I)\right)}-\alpha\right)^{+}$. Moreover we have

$$
\bar{u}^{\prime}(x)=-\frac{\chi_{\Omega_{c}} \bar{p}(x)}{\alpha+\bar{\lambda}} \quad \text { in } L^{1}\left(\left(\Omega_{c},|\bar{u}|\right), L^{2}(I)\right) .
$$

2. Viscous case $\gamma>0$ :

$$
\begin{aligned}
\operatorname{supp}|\bar{u}| & \subseteq\left\{x \in \Omega_{c}:\|\bar{p}\|_{L^{2}(I)}=\alpha\right\}, \\
\bar{u}^{\prime}(x) & =-\frac{\chi_{\Omega_{c}} \bar{p}(x)}{\alpha} \quad \text { in } L^{1}\left(\left(\Omega_{c},|\bar{u}|\right), L^{2}(I)\right) .
\end{aligned}
$$

Proof. First we consider the case $\gamma>0$. A proof of (5.12) and (5.13) is given in [31]. Then we discuss the case $\gamma=0$, which involves norm-constraints on the control. Using $\bar{u} \in U_{a d},\|\bar{p}\|_{\mathcal{C}\left(\Omega_{c}, L^{2}(I)\right)}-\bar{\lambda} \leq \alpha$ and (5.8) we can estimate

$$
\begin{aligned}
& \alpha\|\bar{u}\|_{\mathcal{M}\left(\Omega_{c}, L^{2}(I)\right)}=\int_{\Omega_{c}}\left(\bar{u}^{\prime}, \bar{p}\right)_{L^{2}(I)} \mathrm{d}|\bar{u}|-c_{a d} \bar{\lambda} \\
& \leq \int_{\Omega_{c}}\left(\bar{u}^{\prime}, \bar{p}\right)_{L^{2}(I)} \mathrm{d}|\bar{u}|-\bar{\lambda}\|\bar{u}\|_{\mathcal{M}\left(\Omega_{c}, L^{2}(I)\right)} \\
& \leq \int_{\Omega_{c}}\left\|\bar{u}^{\prime}\right\|_{L^{2}(I)}\|\bar{p}\|_{L^{2}(I)} \mathrm{d}|\bar{u}|-\bar{\lambda}\|\bar{u}\|_{\mathcal{M}\left(\Omega_{c}, L^{2}(I)\right)} \\
& \quad \leq\|\bar{u}\|_{\mathcal{M}\left(\Omega_{c}, L^{2}(I)\right)}\left(\|\bar{p}\|_{\mathcal{C}\left(\Omega_{c}, L^{2}(I)\right)}-\bar{\lambda}\right) \leq \alpha\|\bar{u}\|_{\mathcal{M}\left(\Omega_{c}, L^{2}(I)\right)} .
\end{aligned}
$$

Thus the last chain of inequalities holds with equality and therefore we have

$$
\int_{\Omega_{c}}\left(\bar{u}^{\prime}, \bar{p}\right)_{L^{2}(I)}-\left\|\bar{u}^{\prime}\right\|_{L^{2}(I)}\|\bar{p}\|_{L^{2}(I)} \mathrm{d}|\bar{u}|=0
$$

which implies

$$
\bar{u}^{\prime}=-\frac{1}{\|\bar{p}\|_{L^{2}(I)}} \chi_{\Omega_{c}} \bar{p} \quad \text { in } L^{1}\left(\left(\Omega_{c},|\bar{u}|\right), L^{2}(I)\right) .
$$

Moreover we have

$$
\int_{\Omega_{c}}\|\bar{p}\|_{L^{2}(I)}-\bar{\lambda}-\alpha \mathrm{d}|\bar{u}|=0
$$

which then implies (5.10) and (5.11). Equality in (5.14) also implies

$$
\left(c_{a d}-\alpha\|\bar{u}\|_{\mathcal{M}\left(\Omega_{c}, L^{2}(I)\right)}\right) \bar{\lambda}=0 .
$$

Remark 5.21. If the constraint $\|\bar{u}\|_{\mathcal{M}\left(\Omega_{c}, L^{2}(I)\right)} \leq c_{a d}$ is not active, there holds $\bar{\lambda}=0$ and we recover the usual optimality conditions (5.12) and (5.13). This can be guaranteed for a large enough parameter $\alpha$; cf. Remark 4.13.

Copyright $@$ by SIAM. Unauthorized reproduction of this article is prohibited. 
6. Well-posedness of the optimal control problem for $\gamma=0$ without norm-constraint on the control. In this section we prove the existence of optimal controls in the nonviscous case $\gamma=0$ without norm-constraints on the optimal control. The proof is based on the knowledge that the boundedness of the state $y$ in the $\left.L^{4}(I \times \Omega)\right)$-norm implies an a priori estimate of $y$ in $\mathcal{B}$. Therefore, we add the $L^{4}(I \times \Omega)$ norm as an additional regularization term to objective functional of $(\mathcal{P})$ and use the nonstate reduced approach from [35]. In [35], the state equation is considered as an equality constraint in the pair $(y, u)$ in contrast to the reduced approach from the previous sections.

In the following we consider an optimal control problem of the form

$$
\begin{aligned}
\min _{u \in \mathcal{M}\left(\Omega_{c}, L^{2}(I)\right), y \in Y} J(y, u)= & \frac{1}{2}\left(\left\|\chi_{\Omega_{o}} y-z_{1}\right\|_{L^{2}\left(I \times \Omega_{o}\right)}^{2}+\left\|\chi_{\Omega_{o}} y(T)-z_{2}\right\|_{L^{2}\left(\Omega_{o}\right)}^{2}\right) \\
& +\alpha\|u\|_{\mathcal{M}\left(\Omega, L^{2}(I)\right)}+\frac{\eta}{4}\|y\|_{L^{4}(I \times \Omega)}^{4}
\end{aligned}
$$

subject to (1.2a)-(1.2c) with $\gamma=0$.

Next we introduce the notion of a state-control pair.

Definition 6.22. Let $(y, u) \in Y \times \mathcal{M}\left(\Omega_{c}, L^{2}(I)\right)$ such that $y(T, \cdot) \in L^{2}(\Omega)$. They are called a state-control pair if they satisfy the equation

$$
\int_{0}^{T}(y, \phi)_{L^{2}(\Omega)} \mathrm{d} t+\left(y(T), p_{T}\right)_{L^{2}(\Omega)}=\int_{0}^{T}\left\langle u-y \partial_{x} y, p\right\rangle_{H^{-1}(\Omega), H_{0}^{1}(\Omega)} \mathrm{d} t+\left(y_{0}, p(0)\right)_{L^{2}(\Omega)}
$$

for all $\left(\phi, p_{T}\right) \in L^{1}\left(I, L^{2}(\Omega)\right) \times L^{2}(\Omega)$, where $p=p\left(\phi, p_{T}\right) \in \mathcal{B}$ is the mild solution of $(3.2 \mathrm{a})-(3.2 \mathrm{c})$.

Now we proof the existence of an optimal state-control pair.

Proposition 6.23. There exists a global solution $(\bar{y}, \bar{u}) \in Y \times \mathcal{M}\left(\Omega_{c}, L^{2}(I)\right)$ to the optimal control problem (6.2).

Proof. Since the cost functional $J$ is positive it has an infimum $\bar{J}$. Moreover we already have shown in Proposition 3.7 existence of a control-state pair $(y, u) \in$ $Y \times \mathcal{M}\left(\Omega_{c}, L^{2}(I)\right)$. (For small data, see subsection 3.3.) Thus there exists a minimizing sequence of state-control pairs $\left(y_{n}, u_{n}\right) \in Y \times \mathcal{M}\left(\Omega_{c}, L^{2}(I)\right)$ such that $J\left(y_{n}, u_{n}\right) \rightarrow \bar{J}$ as $n \rightarrow \infty$. Furthermore there is a $\varepsilon>0$ such that

$$
\varepsilon+J(\hat{y}, 0) \geq \alpha\left\|u_{n}\right\|_{\mathcal{M}\left(\Omega_{c}, L^{2}(I)\right)}+\frac{\eta}{4}\left\|y_{n}\right\|_{L^{4}(I \times \Omega)}^{4}
$$

for $n$ large enough and $\hat{y}$ is the solution of (1.2a)-(1.2c) for $u=0$. Therefore the sequence $\left(y_{n}, u_{n}\right)$ is bounded in $L^{4}(I \times \Omega) \times \mathcal{M}\left(\Omega_{c}, L^{2}(I)\right)$. Thanks to (6.4), the sequence of control-state pairs satisfies the estimate

$$
\begin{array}{rl}
\left\|y_{n}\right\|_{\mathcal{B}}+\left\|\partial_{t} y_{n}\right\|_{L^{2}\left(I, \mathcal{V}^{*}\right)} & \leq C(T, L)\left(\left\|u_{n}\right\|_{\mathcal{M}\left(\Omega_{c}, L^{2}(I)\right)}+\left\|y_{0}\right\|_{L^{2}(\Omega)}\right. \\
& \left.+\left\|y_{n} \partial_{x} y_{n}\right\|_{L^{2}\left(I, H^{-1}(\Omega)\right)}\right) \\
\leq C & C(T, L)\left(\left\|u_{n}\right\|_{\mathcal{M}\left(\Omega_{c}, L^{2}(I)\right)}+\left\|y_{0}\right\|_{L^{2}(\Omega)}+\left\|y_{n}\right\|_{L^{4}(I \times \Omega)}^{2}\right)
\end{array}
$$

according to (3.5) and the proof of Lemma 3.6. Thus we also have that $y_{n}$ is bounded in $Y$. This implies the existence of an element $(\bar{y}, \bar{u}) \in Y \times \mathcal{M}\left(\Omega_{c}, L^{2}(I)\right)$ and a state-control subsequence $\left(y_{n_{k}}, u_{n_{k}}\right)$ such that $u_{n_{k}}$ converges in the weak-* topology 
of $\mathcal{M}\left(\Omega_{c}, L^{2}(I)\right)$ toward $\bar{u}$ and $y_{n_{k}}$ converges in the weak-* topology of $Y$ toward $\bar{y}$. Moreover the convergence $y_{n_{k}}(T) \rightarrow \bar{y}(T)$ in $L^{2}(\Omega)$ is ensured. With the Aubin-Lions lemma, we also get

$$
y_{n_{k}} \rightarrow \bar{y} \quad \text { in } \quad L^{2}\left(I, \mathcal{C}_{0}(\Omega)\right)
$$

Next we check that $(\bar{y}, \bar{u})$ is a state-control pair according to Definition 6.22 . The convergence of the linear terms in (6.2) is obvious. The convergence of the nonlinear terms is due to strong convergence of $y_{n_{k}}$ toward $\bar{y}$. Therefore $(\bar{y}, \bar{u})$ is a state-control pair and satisfies the weak formulation (6.2). The tracking functional (in which we include the $L^{4}$ part) is weakly lower semicontinuous in $L^{4}(I \times \Omega) \times L^{2}(\Omega)$, and the control term is weakly-* lower semicontinuous in $\mathcal{M}\left(\Omega_{c}, L^{2}(I)\right)$. Therefore the statecontrol pair $(\bar{y}, \bar{u})$ is a minimizer of $J$.

7. Discretization and numerical results. This section is devoted to the discretization of the optimization problem $(\mathcal{P})$ as well as some insights into the numerical optimization process we use.

7.1. Discretization of state and adjoint equation. In the case of bounded domains numerous schemes are available, for example, finite difference schemes [21, 55], finite element schemes [53, 1], finite volume schemes [22], discontinuous Galerkin schemes $[5,54]$, or polynomial spectral methods [36, 37, 46]. One of the most recent and efficient method for the $\mathrm{KdV}$ is proposed in [36]. The linear term is treated by a Petrov-Galerkin method on the Legendre polynomials, whereas the nonlinear term is treated pseudospectrally on the Chebyschev collocation points. Shortly after, Shen [46] proposed an improvement of this Petrov-Galerkin method with nearly optimal computational complexity. This will be our method of choice and we recall it here briefly. Since it has never been used on optimal control problems, we also discuss an appropriate time discretization which allows the derivation of a consistent adjoint time stepping.

7.1.1. The dual Petrov-Galerkin method. The interesting feature of this method lies in the choice of the test and trial functions bases. They are chosen as a compact (adjacent orders) combination of Legendre polynomials such that the trial functions satisfy the underlying boundary conditions of (3.1b) and the test functions satisfy the dual boundary conditions (3.2b). Therefore, most matrices, i.e., mass matrices and stiffness matrices, involved are sparse or well-conditioned; cf. [46]. We present the method on some reference domain $[-1,1]$, but it can be extended to any other domain of type $[a, b]$ by scaling the Legendre polynomials and the integrals. Throughout the following sections we assume $\gamma \geq 0$, i.e., all discussed results apply in the viscous as well as the nonviscous case. Then we denote by $P_{N}$ the space of polynomials of degree $\leq N$ and set

$$
\begin{gathered}
V_{N}=\operatorname{span}\left\{y \in P_{N}: y(1)=y(-1)=\partial_{x} y(1)=0\right\}, \\
V_{N}^{*}=\operatorname{span}\left\{y \in P_{N}: y(1)=y(-1)=\partial_{x} y(-1)=0\right\} .
\end{gathered}
$$

We consider then the semidiscrete problem: Find

$$
y_{N}: I \rightarrow V_{N}, \quad t \mapsto y_{N}(t, \cdot),
$$



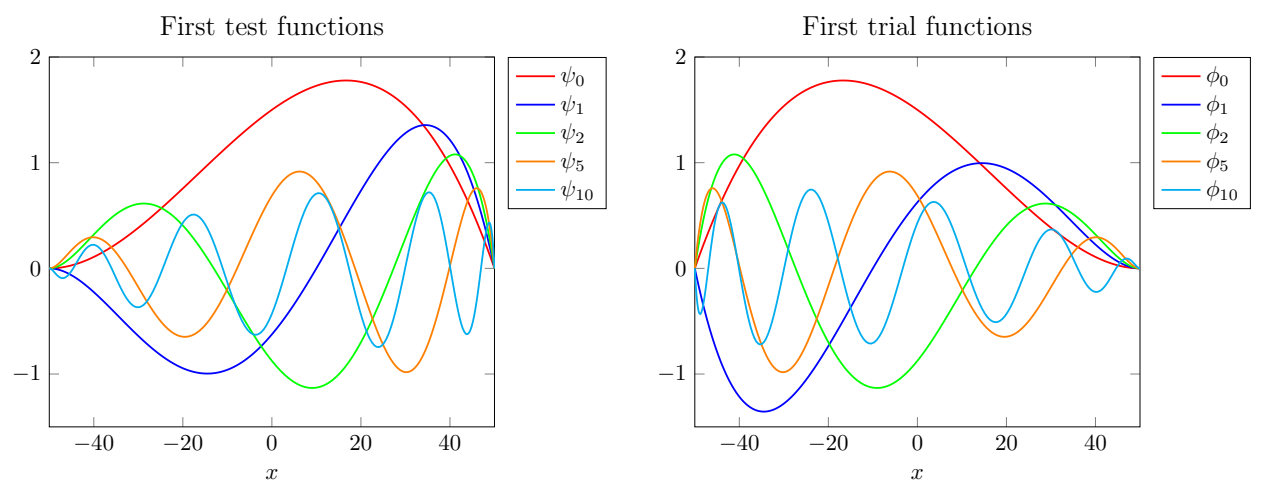

Fig. 1. First test and trial functions.

such that for almost every $t \in I$

$$
\begin{aligned}
& \left(\partial_{t} y_{N}, \varphi_{N}\right)+\left(\partial_{x} y_{N}, \varphi_{N}\right)+\left(\partial_{x} y_{N}, \partial_{x x} \varphi_{N}\right) \\
+ & \gamma\left(\partial_{x} y_{N}, \partial_{x} \varphi_{N}\right)-\left(\frac{y_{N}^{2}}{2}, \partial_{x} \varphi_{N}\right)=\left\langle u, \varphi_{N}\right\rangle_{\mathcal{M}\left(\Omega_{c}, L^{2}(I)\right), \mathcal{C}\left(\Omega_{c}, L^{2}(I)\right)} \forall \varphi_{N} \in V_{N}^{*}
\end{aligned}
$$

holds where $(\cdot, \cdot)$ denotes the usual $L^{2}(\Omega)$-inner product. Denoting by $L_{k}$ the $k$ th Legendre polynomial, we can define the basis functions as follows (see Figure 1):

$$
\begin{aligned}
& \phi_{k}(x)=L_{k}(x)-\frac{2 k+3}{2 k+5} L_{k+1}(x)-L_{k+2}(x)+\frac{2 k+3}{2 k+5} L_{k+3}(x), \\
& \psi_{k}(x)=L_{k}(x)+\frac{2 k+3}{2 k+5} L_{k+1}(x)-L_{k+2}(x)-\frac{2 k+3}{2 k+5} L_{k+3}(x) .
\end{aligned}
$$

Thus for $N \geq 3$, we have

$$
\begin{aligned}
V_{N} & =\operatorname{span}\left\{\phi_{0}, \phi_{1}, \ldots, \phi_{N-3}\right\}, \\
V_{N}^{*} & =\operatorname{span}\left\{\psi_{0}, \psi_{1}, \ldots, \psi_{N-3}\right\} .
\end{aligned}
$$

Then, we introduce the semidiscrete state variable $y_{N}(t, \cdot)$ on spectral space and its vector representation:

$$
y_{N}(t, \cdot)=\sum_{k=0}^{N-3} \hat{y}_{k}(t) \phi_{k}(\cdot), \quad \mathbf{y}(t)=\left(\hat{y}_{0}(t), \hat{y}_{1}(t), \ldots, \hat{y}_{N-3}(t)\right)^{T} .
$$

The vector representation of the control is given by

$$
\mathbf{u}(t)=\left(\left\langle u_{N}(t, \cdot), \psi_{0}(\cdot)\right\rangle,\left\langle u_{N}(t, \cdot), \psi_{1}(\cdot)\right\rangle, \ldots,\left\langle u_{N}(t, \cdot), \psi_{N-3}(\cdot)\right\rangle\right)^{T},
$$

where the form of the semidiscrete control $u_{N}(t, \cdot)$ is given in subsection 7.1.2. Based on these discrete spaces, we build the matrices $M, P, Q$, and $S$ of size $(N-2) \times(N-2)$ with the coefficients $m_{i j}, p_{i j}, q_{i j}$, and $s_{i j}$ defined as follows:

$$
m_{i j}=\left(\phi_{j}, \psi_{i}\right), \quad p_{i j}=\left(\partial_{x} \phi_{j}, \psi_{i}\right), \quad q_{i j}=\left(\partial_{x} \phi_{j}, \partial_{x} \psi_{i}\right), \quad s_{i j}=\left(\partial_{x} \phi_{j}, \partial_{x x} \psi_{i}\right)
$$

Copyright $@$ by SIAM. Unauthorized reproduction of this article is prohibited. 
The variational formulation (7.3) thus yields

$$
M \frac{d \mathbf{y}}{d t}+(P+\gamma Q+S) \mathbf{y}+F(\mathbf{y})=\mathbf{u},
$$

where $F(\mathbf{y})$ is the nonlinear term and is treated as suggested in [46], i.e., using the pseudospectral approach. This means that the nonlinearity is evaluated in the spatial domain given by Chebyshev-Gauss-Lobatto (CGL) points and is transferred back in the Legendre spectral space. Therefore, we need to be able to transform back and forth from the spectral space of Legendre coefficients to the values in the CGL points. This can be done using the fast Fourier transform (FFT) and the Chebyshev-Legendre transform. However, for the polynomial degrees we consider here (between 160 and 512), we use a simpler and more direct method which is faster and allows for an easier derivation of the discrete adjoint state equation (see subsection 7.1.3). It consists of building the matrices $L_{1}=\left(\phi_{j}\left(x_{i}\right)\right)$ and $L_{2}=\left(\psi_{j}\left(x_{i}\right)\right), i=0 \ldots N, j=0 \ldots N-3$ beforehand, where the points $x_{i}$ are the CGL points. By construction, those matrices satisfy

$$
L_{1} \mathbf{y}(t)=\tilde{\mathbf{y}}(t)=\left(y_{N}\left(t, x_{0}\right), y_{N}\left(t, x_{2}\right), \ldots, y_{N}\left(t, x_{N}\right)\right)^{T},
$$

and the same holds true for a variable from $V_{N}^{*}$ with $L_{2}$. Thus the evaluation of the nonlinear term is given by

$$
L_{1} F(\mathbf{y}(t))=\tilde{\mathbf{y}}^{2}(t)=\left(y_{N}^{2}\left(t, x_{0}\right), y_{N}^{2}\left(t, x_{2}\right), \ldots, y_{N}^{2}\left(t, x_{N}\right)\right)^{T} .
$$

Note that the spatial space, in which $\tilde{\mathbf{y}}(t)$ lies, and the spectral space, in which $\mathbf{y}(t)$ lies, do not have the same dimensions, i.e., $N+1$ versus $N-2$. This is only caused by the fact that the three boundary conditions are already included in the spectral space and not in the spatial space.

7.1.2. Semidiscretization of the control $\boldsymbol{u}$. By $\left\{x_{i}\right\}, i=0,1, \ldots, N$, we denote the grid points of the spatial mesh (i.e., in our case the CGL points). The control is then discretized as follows:

$$
u_{N}(t, \cdot)=\sum_{j=0}^{N} \hat{u}_{j}(t) \delta_{x_{j}}(\cdot),
$$

where $\delta_{x_{j}}$ are Dirac functionals located at the grid points $x_{j}$ and the functions $\hat{u}_{j}(t)$ are its time-dependent coefficients. Hence, there holds $u_{N} \in \mathcal{M}\left(\Omega_{c}, L^{2}(I)\right)$. Thus the right-hand side of (7.3) takes the form

$$
\left\langle u_{N}(t), \psi\right\rangle=\sum_{j=0}^{N} \hat{u}_{j}(t) \psi\left(x_{j}\right) .
$$

Moreover we introduce another vectorized representation of the variable $u_{N}$

$$
\hat{\mathbf{u}}(t)=\left(\hat{u}_{0}(t), \ldots, \hat{u}_{N}(t)\right)^{T} .
$$

This representation of the discrete control will be useful in the definition of the various norms involved in the optimization process. 
7.1.3. Time-stepping scheme and adjoint-Crank-Nicolson-leap-frog. The KdVB equation involves a high order derivative. Therefore an explicit temporal discretization would lead to small time steps in order to get stability. Thus an implicit method seems to be more more appropriate. In [34], the authors prove convergence of a pseudospectral method in space combined with a backward Euler scheme for the KdV equation. Due the first order convergence of this method, small time steps are required to achieve a good level of accuracy. Therefore, a second order implicit scheme like the Crank-Nicolson scheme is preferable. This scheme is well studied in the context of optimal control of PDEs. Using the representation of the Crank-Nicolson scheme as a Petrov-Galerkin method with continuous linear trial functions and discontinuous piecewise constant test functions determines the form of the discrete adjoint, tangent, and additional adjoint equations. The solutions of these discrete equations are used for the exact computation of the discrete gradient and Hessian, e.g., [38]. Note that the use of the exact discrete derivatives is important for the convergence of optimization algorithms. However, the Crank-Nicolson scheme is computationally demanding. An alternative is the two-step CNLF method in which the third derivative is treated implicitly and the nonlinear term is treated explicitly. This method has already been extensively used for the KdV equation; cf. [46, 36, 37]. Commonly, it is initialized by a semi-implicit step. We suggest also a slight modification of the last step of this two-step method in order to get a discrete adjoint state equation that is consistent with the continuous adjoint state equation.

The time domain is divided into $N_{T}$ intervals, i.e., $0=t_{0}<t_{1}, \ldots,<t_{N_{T}}=T$. The first and last one have length $\frac{\Delta t}{2}$ and the other steps are of equal length $\Delta t$. The fully discrete state variable has the form

$$
\mathbf{y}_{n}=\mathbf{y}\left(t_{n}\right)=\left(\hat{y}_{0}\left(t_{n}\right), \hat{y}_{1}\left(t_{n}\right), \ldots, \hat{y}_{N-3}\left(t_{n}\right)\right)^{T}, \quad n=0, \ldots, N_{T}
$$

and the control

$$
\begin{aligned}
& \mathbf{u}_{n}=\mathbf{u}\left(t_{n}\right)=\left(\left\langle u_{N}\left(t_{n}, \cdot\right), \psi_{0}(\cdot)\right\rangle,\left\langle u_{N}\left(t_{n}, \cdot\right), \psi_{1}(\cdot)\right\rangle, \ldots,\left\langle u_{N}\left(t_{n}, \cdot\right), \psi_{N-3}(\cdot)\right\rangle\right)^{T} \\
& \hat{\mathbf{u}}_{n}=\hat{\mathbf{u}}\left(t_{n}\right)=\left(\hat{u}_{0}\left(t_{n}\right), \ldots, \hat{u}_{N}\left(t_{n}\right)\right)^{T}, \quad n=0, \ldots, N_{T}
\end{aligned}
$$

where the duality pairing $\langle\cdot, \cdot\rangle$ is evaluated according to (7.14). With the help of the matrices defined in (7.9), the forward scheme looks as follows:

$$
\left\{\begin{aligned}
& \frac{1}{2}(M+\Delta t S) \mathbf{y}_{1}=\frac{1}{2}(M+\Delta t(P+\gamma Q)) \mathbf{y}_{0} \\
&+\frac{1}{2} \Delta t F\left(\mathbf{y}_{0}\right)+\frac{1}{2} \Delta t \mathbf{u}_{0}, \\
& \frac{1}{2}(M+\Delta t S) \mathbf{y}_{n+1}=\frac{1}{2}(M-\Delta t S) \mathbf{y}_{n-1}+ \Delta t(P+\gamma Q) \mathbf{y}_{n} \\
&+\Delta t F\left(\mathbf{y}_{n}\right)+\Delta t \mathbf{u}_{n}, n=1, \ldots, N_{T}-1 \\
& M \mathbf{y}_{N_{T}+1}=\frac{1}{2}\left(M \mathbf{y}_{N_{T}}+M \mathbf{y}_{N_{T}-1}\right)+\frac{1}{2} \Delta t(P+\gamma Q) \mathbf{y}_{N_{T}} \\
&+\frac{1}{2} \Delta t F\left(\mathbf{y}_{N_{T}}\right)-\frac{1}{2} \Delta t S \mathbf{y}_{N_{T}-1} .
\end{aligned}\right.
$$

The first step is semi-implicit. The steps $n=1, \ldots, N_{T}-1$ are classical CNLF steps. The last step is not a regular step. It is designed in a way such that the discrete adjoint state equation is consistent with a CNLF discretization of the continuous adjoint state equation. Notice that this step becomes the mean value between the two preceding 
steps for $\Delta t \downarrow 0$. The corresponding discrete objective functional has the form

$$
\begin{aligned}
J_{N, N_{T}}\left(\mathbf{y}_{0}, \ldots, \mathbf{y}_{N_{T}},\right. & \left.\mathbf{u}_{0}, \ldots, \mathbf{u}_{N_{T}}\right)=\frac{1}{2} \sum_{i=1}^{N_{T}} \Delta t\left(\mathbf{y}_{i}-\mathbf{z}_{1, i}\right)^{t} A\left(\mathbf{y}_{i}-\mathbf{z}_{1, i}\right) \\
+ & \frac{1}{2}\left(\mathbf{y}_{N_{T}}-\mathbf{z}_{2}\right)^{t} A\left(\mathbf{y}_{N_{T}}-\mathbf{z}_{2}\right)+\alpha \sum_{j=1}^{N}\left(\sum_{i=1}^{N_{T}} \Delta t \hat{\mathbf{u}}_{i j}^{2}\right)^{1 / 2}
\end{aligned}
$$

where entries of $A$ are given by $a_{i j}=\left(\chi_{\Omega_{o}} \phi_{j}, \psi_{i}\right)_{L^{2}(\Omega)}$. Then we define the discrete Lagrangian function:

$$
\begin{gathered}
\mathcal{L}_{N, N_{T}}\left(\mathbf{y}_{0}, \ldots, \mathbf{y}_{N_{T}}, \mathbf{u}_{0}, \ldots, \mathbf{u}_{N_{T}}, \mathbf{p}_{0}, \ldots, \mathbf{p}_{N_{T}-1}\right) \\
=J_{N, N_{T}}\left(\mathbf{y}_{0}, \ldots, \mathbf{y}_{N_{T}}, \mathbf{u}_{0}, \ldots, \mathbf{u}_{N_{T}}\right) \\
+\mathbf{p}_{0}^{T}\left[\frac{1}{2}(M+\Delta t S) \mathbf{y}_{1}-\frac{1}{2}(M+\Delta t P+\gamma \Delta t Q) \mathbf{y}_{0}-\frac{1}{2} \Delta t F\left(\mathbf{y}_{0}\right)-\frac{1}{2} \Delta t \mathbf{u}_{0}\right] \\
+\sum_{k=1}^{N_{T}-2} \mathbf{p}_{k}^{T}\left[\frac{1}{2}(M+\Delta t S) \mathbf{y}_{k+1}-\frac{1}{2}(M-\Delta t S) \mathbf{y}_{k-1}-\Delta t(P+\gamma Q) y_{k}\right. \\
\left.-\Delta t F\left(y_{k}\right)-\Delta t \mathbf{u}_{k}\right] \\
+\mathbf{p}_{N_{T}-1}^{T}\left[M \mathbf{y}_{N_{T}}-\frac{1}{2}\left(M \mathbf{y}_{N_{T}-1}+M \mathbf{y}_{N_{T}-2}\right)-\frac{1}{2} \Delta t(P+\gamma Q) \mathbf{y}_{N_{T}-1}\right. \\
\left.\quad-\frac{1}{2} \Delta t F\left(\mathbf{y}_{N_{T}-1}\right)+\frac{1}{2} \Delta t S \mathbf{y}_{N_{T}-2}\right] .
\end{gathered}
$$

From (7.19), we can derive the discrete adjoint state equation which has the form

$$
\left\{\begin{aligned}
M^{T} \mathbf{p}_{N_{T}-1}=-A\left(\mathbf{y}_{N_{T}}-\mathbf{z}_{2}\right) & \\
\frac{1}{2}\left(M^{T}+\Delta t S^{T}\right) \mathbf{p}_{N_{T}-2}= & \frac{1}{2}\left(M^{T}+\Delta t P^{T}+\gamma \Delta t Q^{T}\right) \mathbf{p}_{N_{T}-1} \\
& \quad+\frac{1}{2} \Delta t F^{\prime}\left(\mathbf{y}_{N_{T}-1}\right)^{T} \mathbf{p}_{N_{T}-1} \\
\frac{1}{2}\left(M^{T}+\Delta t S^{T}\right) \mathbf{p}_{n-2}= & \frac{1}{2}\left(M^{T}-\Delta t S^{T}\right) \mathbf{p}_{n}+\Delta t\left(P^{T}+\gamma Q^{T}\right) \mathbf{p}_{n-1} \\
& +\Delta t F^{\prime}\left(\mathbf{y}_{n-1}\right)^{T} \mathbf{p}_{n-1}, \quad n=2, \ldots, N_{T}-1
\end{aligned}\right.
$$

in the case of terminal observations, which will be of particular interest in the numerical examples. In the first equation the terminal value of the discrete adjoint state is set. This is followed by a single semi-implicit step and regular CNLF steps.

Remark 7.24. Without this modification of the last step in (7.17), the discrete adjoint state equation obtained in the case of terminal observation would be

$$
\left\{\begin{aligned}
\frac{1}{2}\left(M^{T}+\Delta t S^{T}\right) \mathbf{p}_{N_{T}-1}=-A\left(\mathbf{y}_{N_{T}}-\mathbf{z}_{2}\right) \\
\frac{1}{2}\left(M^{T}+\Delta t S^{T}\right) \mathbf{p}_{N_{T}-2}=\left(\Delta t P^{T}+\gamma \Delta t Q^{T}\right) \mathbf{p}_{N_{T}-1}+\Delta t F^{\prime}\left(\mathbf{y}_{N_{T}-1}\right)^{T} \mathbf{p}_{N_{T}-1} \\
\frac{1}{2}\left(M^{T}+\Delta t S^{T}\right) \mathbf{p}_{n-2}=\frac{1}{2}\left(M^{T}-\Delta t S^{T}\right) \mathbf{p}_{n}+\Delta t\left(P^{T}+\gamma Q^{T}\right) \mathbf{p}_{n-1} \\
\quad+\Delta t F^{\prime}\left(\mathbf{y}_{n-1}\right)^{T} \mathbf{p}_{n-1}, \quad n=2, \ldots, N_{T}-1 .
\end{aligned}\right.
$$

Copyright $@$ by SIAM. Unauthorized reproduction of this article is prohibited. 
The major difference is the second step. It does not correspond to a consistent discretization of the continuous adjoint state equation. The time derivative is not reconstructed. In practice this would result in large oscillations in time of the adjoint variable.

Remark 7.25. Unlike the other steps, the modified last step in (7.17) involves the inversion of the matrix $M$, which is typically ill-conditioned. Therefore, we invert the matrix $M+\varepsilon I d$ with a $\varepsilon$ of the order $h^{2}$, where $h$ is the largest space step.

Proposition 7.26. The directional derivative of

$$
\left\{\mathbf{u}_{i}\right\}_{i=1}^{N_{T}} \mapsto \frac{1}{2}\left(\mathbf{y}_{N_{T}}-\mathbf{z}_{2}\right)^{t} A\left(\mathbf{y}_{N_{T}}-\mathbf{z}_{2}\right),
$$

where $\left\{\mathbf{y}_{i}\right\}_{i=1}^{N_{T}}$ is the solution of (7.17), is given by $\left\{-\mathbf{p}_{i}\right\}_{i=1}^{N_{T}}$, where $\left\{\mathbf{p}_{i}\right\}_{i=1}^{N_{T}}$ is the solution of (7.20).

Proof. The proof is done by standard differentiation of the discrete Lagrangian.

7.2. Numerical treatment of the optimization problem. In this section we consider the optimal control problem $(\mathcal{P})$ in both the viscous and nonviscous case, i.e., $\gamma \geq 0$, without additional norm-constraints on the control. Thus we have $U_{a d}=\mathcal{M}\left(\Omega_{c}, L^{2}(I)\right)$.

7.2.1. The regularized problem. In this section, we discuss the solution of the optimal control problem $(\mathcal{P})$ by a Newton type method and a continuation strategy. We introduce an additional $L^{2}$ regularization term in $(\mathcal{P})$ :

$$
\begin{array}{r}
\min _{u \in L^{2}\left(\Omega_{c} \times I\right), y \in Y} J(y, u)=\frac{1}{2}\left(\left\|\chi_{\Omega_{o}} y-z_{1}\right\|_{L^{2}\left(I \times \Omega_{o}\right)}^{2}+\left\|\chi_{\Omega_{o}} y(T)-z_{2}\right\|_{L^{2}\left(\Omega_{o}\right)}^{2}\right) \\
+\alpha\|u\|_{L^{1}\left(\Omega_{c}, L^{2}(I)\right)}+\frac{\varepsilon}{2}\|u\|_{L^{2}\left(I \times \Omega_{c}\right)}^{2} \quad \text { s.t. }(1.2 \mathrm{a})-(1.2 \mathrm{c}) .
\end{array}
$$

This problem is posed in the Hilbert space $L^{2}\left(I \times \Omega_{c}\right)$. Since the embedding $L^{1}\left(\Omega_{c}, L^{2}(I)\right) \hookrightarrow \mathcal{M}\left(\Omega_{c}, L^{2}(I)\right)$ is isometric, we have

$$
\psi(u)=\|u\|_{\mathcal{M}\left(\Omega_{c}, L^{2}(I)\right)}=\|u\|_{L^{1}\left(I, L^{2}\left(\Omega_{c}\right)\right)}=\int_{\Omega_{c}}\|u(x)\|_{L^{2}(I)} d x
$$

for any $L^{2}\left(I \times \Omega_{c}\right)$. In [27], the authors study this problem in the linear parabolic case and prove that this setting promotes a striped sparsity pattern of the optimal control. For the derivation of optimality conditions, we introduce the proximal map of the $L^{1}\left(\Omega_{c}, L^{2}(I)\right)$ norm, e.g., [2]:

$$
\operatorname{Prox}_{\psi / \varepsilon}(q)(t, x)=\left(1-\frac{\alpha}{\varepsilon\|q(x)\|_{L^{2}(I)}}\right)^{+} q(t, x) \quad \text { for } q \in L^{2}\left(I \times \Omega_{c}\right) .
$$

We can then express the optimality condition for (7.22) by the pointwise formula

$$
\bar{u}_{\varepsilon}(t, x)=\operatorname{Prox}_{\psi / \varepsilon}\left(-\frac{1}{\varepsilon} \bar{p}_{\varepsilon}\right)(t, x)=-\frac{1}{\varepsilon}\left(1-\frac{\alpha}{\left\|\bar{p}_{\varepsilon}(x)\right\|_{L^{2}(I)}}\right)^{+} \bar{p}_{\varepsilon}(t, x),
$$

where $\bar{p}_{\varepsilon}$ solves (5.4a)-(5.4c) for $\phi=\bar{y}_{\varepsilon}-z_{1}, y=\bar{y}_{\varepsilon}$, and $p_{T}=\bar{y}_{\varepsilon}(T)-z_{2}$ and $\bar{y}_{\varepsilon}$ solves (1.2a)-(1.2c) for $\bar{u}$. Now a Newton type method can applied for the solution of

$$
F\left(u_{\varepsilon}\right)(t, x)=\varepsilon u_{\varepsilon}(t, x)+\left(1-\frac{\alpha}{\left\|p_{\varepsilon}(x)\right\|_{L^{2}(I)}}\right)^{+} p_{\varepsilon}(t, x)=0 .
$$

Copyright (C) by SIAM. Unauthorized reproduction of this article is prohibited. 
However, we follow [43] and reformulate the optimality condition (7.26) using the "normal map" due to Robinson. For that we introduce the auxiliary variable $q_{\varepsilon}:=$ $-p_{\varepsilon} / \varepsilon$, where $p_{\varepsilon}$ solves (5.4a)-(5.4c) for $\phi=y_{\varepsilon}-z_{1}, y=y_{\varepsilon}$, and $p_{T}=y_{\varepsilon}(T)-z_{2}$ and $y_{\varepsilon}$ solves (1.2a)-(1.2c) for $u=\operatorname{Prox}_{\psi / \varepsilon}\left(q_{\varepsilon}\right)$. Thus we solve the equation

$$
\mathrm{G}\left(q_{\varepsilon}\right)=\varepsilon q_{\varepsilon}+p_{\varepsilon}=0 .
$$

The control can be recovered from the $q_{\varepsilon}$ using the operator $\operatorname{Prox}_{\psi / \varepsilon}$, i.e., $u=$ $\operatorname{Prox}_{\psi / \varepsilon}\left(q_{\varepsilon}\right)$. For the solution of problem (7.27), we apply a Newton type method, and since the proximal operator is not differentiable, we use the concept of semismoothness to derive a generalized derivative for G; cf. [52]. To be more precise, we have

$$
\operatorname{DG}\left(q_{\varepsilon}\right) \delta q=\varepsilon \delta q+\delta p, \quad \delta q \in L^{2}\left(I \times \Omega_{c}\right)
$$

where $\delta p$ solves (5.4a)-(5.4c) for $\phi=\delta y\left(1+\partial_{x} p_{\varepsilon}\right), y=y_{\varepsilon}$, and $p_{T}=\delta y(T)$ and $\delta y$ solves (5.1a)-(5.1c) for $\delta u=\operatorname{DProx}_{\psi / \varepsilon}\left(q_{\varepsilon}\right) \delta q$ and $y=y_{\varepsilon}$. The generalized derivative of the proximal map is given by

$$
\operatorname{DProx}_{\psi / \varepsilon}\left(q_{\varepsilon}\right) \delta q=\left\{\begin{array}{l}
\left(1-\frac{\alpha}{\varepsilon\left\|q_{\varepsilon}\right\|_{L^{2}(I)}}\right) \delta q+\frac{\alpha}{\varepsilon} \frac{\left(q_{\varepsilon}, \delta q\right)_{L^{2}(I)}}{\left\|q_{\varepsilon}\right\|_{L^{2}(I)}^{3}} q_{\varepsilon} \\
\quad \text { if }\left\|q_{\varepsilon}\right\|_{L^{2}(I)}>\frac{\alpha}{\varepsilon}, \\
0 \quad \text { otherwise. }
\end{array}\right.
$$

The great advantage of the normal map approach is that the Newton operator $D G\left(q_{\varepsilon}\right)$ is symmetric with respect to the scalar product $\left(\cdot, \operatorname{DProx}_{\psi / \varepsilon}\left(q_{\varepsilon}\right) \cdot\right)_{L^{2}\left(I \times \Omega_{c}\right)}$; cf. [30, 43]. Therefore the conjugate gradient method can be used to solve the Newton system. The operator $D F\left(u_{\varepsilon}\right)$ is in general not symmetric. In the case of a linear state equation numerical experiments show that the continuation strategy in $\varepsilon$ is sufficient to achieve global convergence, e.g., [18]. This is not the case for our problem due to the nonlinearity of the state equation. In addition to the continuation strategy in $\varepsilon$ a globalization strategy based on the decrease of the regularized objective functional is required. We implement a trust region semismooth Newton based on the truncated conjugate gradient algorithm by Steihaug [43, 50]. A concise explanation is also available in [30, sections 6.2 and A.3].

7.2.2. Discretization of the control and mass lumping. In the unregularized problem, the controls are discretized as nodal Dirac measures. In the regularized case, this is not possible due to the $L^{2}\left(I \times \Omega_{c}\right)$ regularization term. Thus we introduce a discretization of the control which is compatible to the Dirac discretization in the limiting case $\varepsilon \rightarrow 0$, i.e., we follow the strategy in [31]. The control is discretized in space with piecewise linear and continuous finite elements on a grid whose nodes are given by the CGL-points $\left(x_{n}\right), n=0, \ldots, N$ previously mentioned. Thus, the discrete control has the form

$$
u_{h}=\sum_{j=1}^{N_{T}} \chi_{I_{j}} \sum_{n=0}^{N} \hat{\mathbf{u}}_{j n} e_{n},
$$

where $\chi_{I_{j}}, I_{j}=\left(t_{j-1}, t_{j}\right]$ is the characteristic function of the $j^{t h}$ time interval, and $e_{n}$ is the finite element basis function centered at the grid point $x_{n}$. All scalar products and norms involving the discrete control are computed using mass lumping, i.e., the trapezoidal rule is used for the evaluation of the spatial integrals on each cell. 
Therefore, we have

$$
\begin{aligned}
& \left\|u_{h}\right\|_{L^{1}\left(\Omega, L^{2}(I)\right)}=\sum_{n=0}^{N} d_{n}\left(\sum_{j=1}^{N_{T}} \Delta t \hat{\mathbf{u}}_{n j}^{2}\right)^{1 / 2}, \quad\left\|u_{h}\right\|_{L^{2}\left(\Omega, L^{2}(I)\right)}^{2}=\sum_{n=0}^{N} d_{n}\left(\sum_{j=1}^{N_{T}} \Delta t \hat{\mathbf{u}}_{n j}^{2}\right) \\
& \left\langle u_{h}, \psi\right\rangle_{L^{2}(\Omega)}=\sum_{j=1}^{N_{T}} \chi_{I_{j}} \sum_{n=0}^{N} d_{n} \hat{\mathbf{u}}_{j n} \psi_{n}
\end{aligned}
$$

for all spectral basis functions $\psi$, where $\psi_{n}=\psi\left(x_{n}\right)$ and $d_{n}=\int_{\Omega_{c}} e_{n} \mathrm{~d} x$.

Remark 7.27. Due to the use of (7.30), the formulas (7.26), respectively, (7.27), hold also on the discrete level.

7.3. Numerical examples. In this section, we investigate an inverse problem involving the flow of water in a narrow channel and the problem of the optimal positioning and vertical movement of a wavemaker. A similar optimal design problem is carried out in [41]. A possible application is the design of artificial surfing facilities. For that purpose, we use a version of the forced KdV equation with physical motivated parameters, as in [39]:

$$
\partial_{t} y+f \partial_{x} y-\frac{1}{6} \partial_{x x x} y-\frac{3}{2} y \partial_{x} y=u,
$$

where the parameter $f$ is proportional to $F-1$. ( $F$ is the Froude number.) Its value determines if a flow is subcritical $(f \leq 0)$ or supercritical $(f>0)$. The forcing $u$ is interpreted as the spatial derivative of the bottom topography of the channel. In both examples, we consider the minimization problem

$$
\min _{u \in \mathcal{M}\left(\Omega_{c}, L^{2}(I)\right), y \in Y} J(y, u)=\frac{1}{2}\left\|\chi_{\Omega_{o}} y(T)-z\right\|_{L^{2}\left(\Omega_{o}\right)}^{2}+\alpha\|u\|_{\mathcal{M}\left(\Omega_{c}, L^{2}(I)\right)}
$$

for different $\Omega_{c}, \Omega_{o}$, and $z$, where $y$ is the solution of (7.31). In particular, we emphasize that $U_{a d}=\mathcal{M}\left(\Omega_{c}, L^{2}(I)\right)$ and $\gamma=0$. In all numerical examples, we have never encountered blow ups of $y$.

Inverse problem. In the first example, we reconstruct the time-varying bottom topography which creates water waves in a narrow channel. These waves are observed at final time $\mathrm{T}$. We generate beforehand a wave with the source term

$$
u^{\dagger}(t, x)=\left\{\begin{array}{l}
10 \delta_{\{x=1.5\}}, \text { when } 0<t \leq 2.5 \\
0, \text { when } 2.5 \leq t \leq 5
\end{array}\right.
$$

in a subcritical configuration $(f=-0.5)$. The spatial domain is $\Omega=(-30,30)$ and the time horizon is $T=5$. The space-time grid is parametrized with $N=256$ spatial grid points and a time-step size of $\Delta t=0.01$. The generated wave is displayed in Figure 2. One can see a series of downstream waves (note that the flow enters the domain from the right) and a solitary wave going upstream generated by the bottom topography induced by $u$. Then, white Gaussian noise is added to the $y^{\dagger}$ at final time $T$. The magnitude of the noise is in average 5 percent of the magnitude of the original wave

$$
\frac{\left\|y^{\dagger}-z\right\|_{L^{2}(I \times \Omega)}}{\left\|y^{\dagger}\right\|_{L^{2}(I \times \Omega)}} \approx 0.05
$$



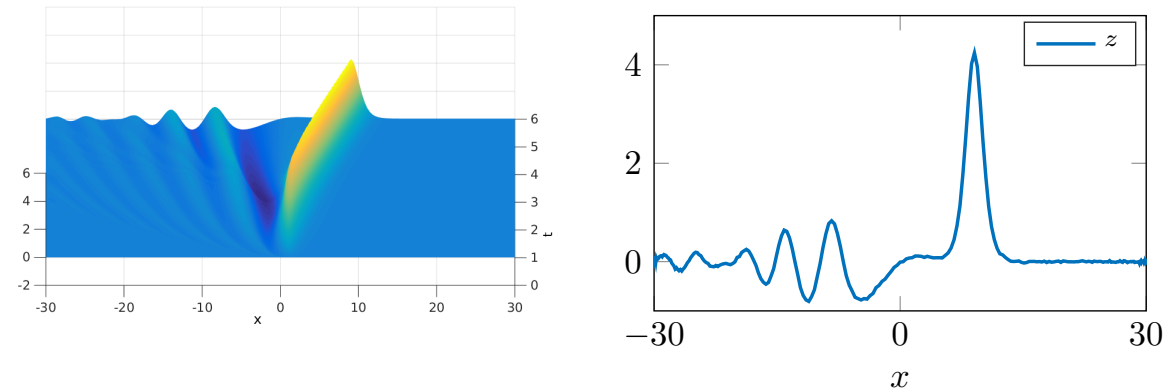

FIG. 2. Left: Exact wave $y^{\dagger}$ generated by the forcing term u. Right: $z$-profile at terminal time with Gaussian white noise.
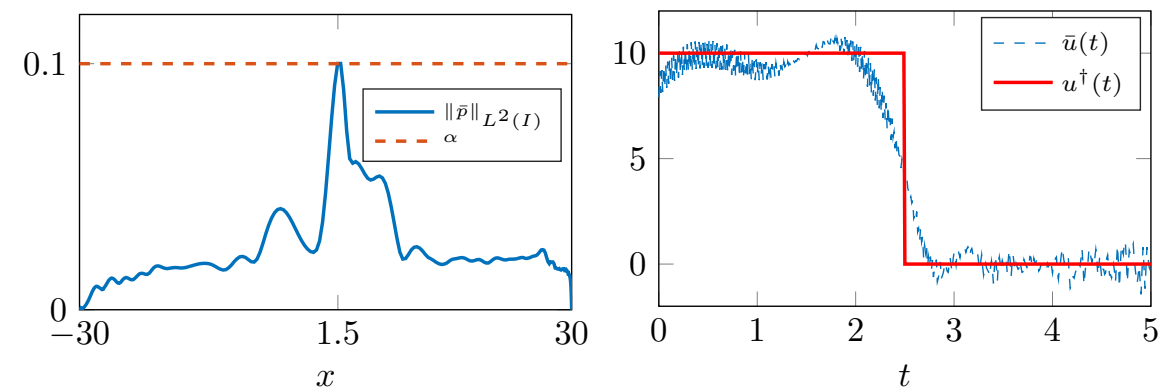

FIG. 3. Left: Spatial support of the control determined by $\|\bar{p}(x)\|_{L^{2}(I)}=\alpha$. Here $\alpha=0.1$ and $\varepsilon=10^{-6}$. Right: Recovered intensity $\bar{u}(t)$ and exact intensity $u^{\dagger}(t)$.
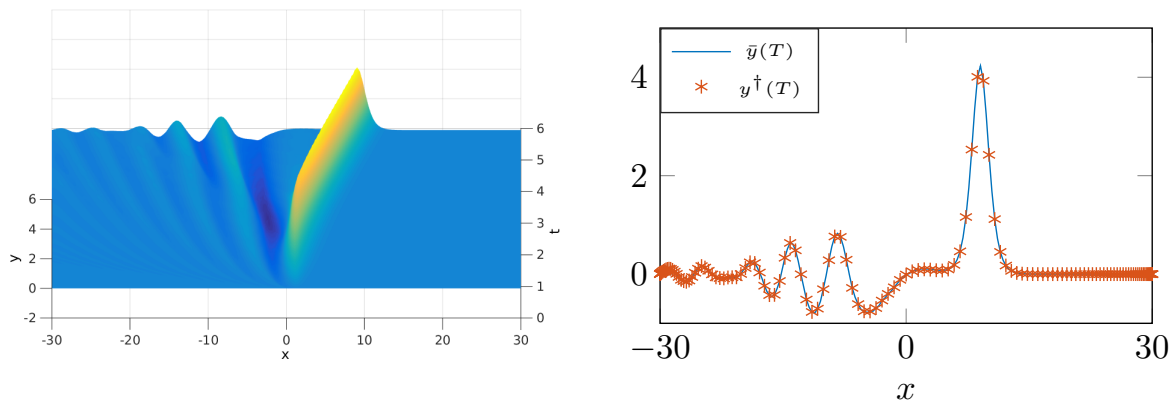

FIG. 4. Recovered state $\bar{y}$ and terminal state $\bar{y}(T)$.

Next, we discuss our numerical results. The support of the optimal control is a subset of the sets where $\|\bar{p}(x)\|_{L^{2}(I)}=\alpha$ holds; see Figure 3 and Proposition 5.20. Thus, the optimal control is a point source, located close to $x=1.5$. The time profile of this point source is depicted in Figure 3 and it follows the original one quite well.

In Figure 4, the recovered state on $I \times \Omega$ and on $\{T\} \times \Omega$ is depicted. We have a quite good tracking of $y^{\dagger}(T)$.

Control example. The second example is a control example which is concerned with the optimal placement of bumps on the bottom of a narrow channel for the generation of a desired flow. We divide the domain $\Omega=(-L, L), L=30$ into two subdomains. The domain $\Omega_{c}=(-L, 0)$ is the control domain, while $\Omega_{o}=(0, L)$ is 

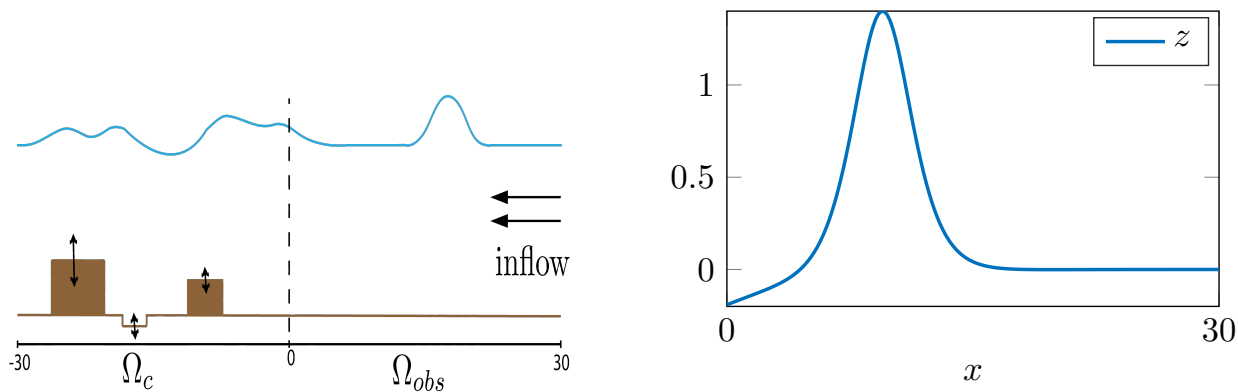

FIG. 5. Control setup and data.
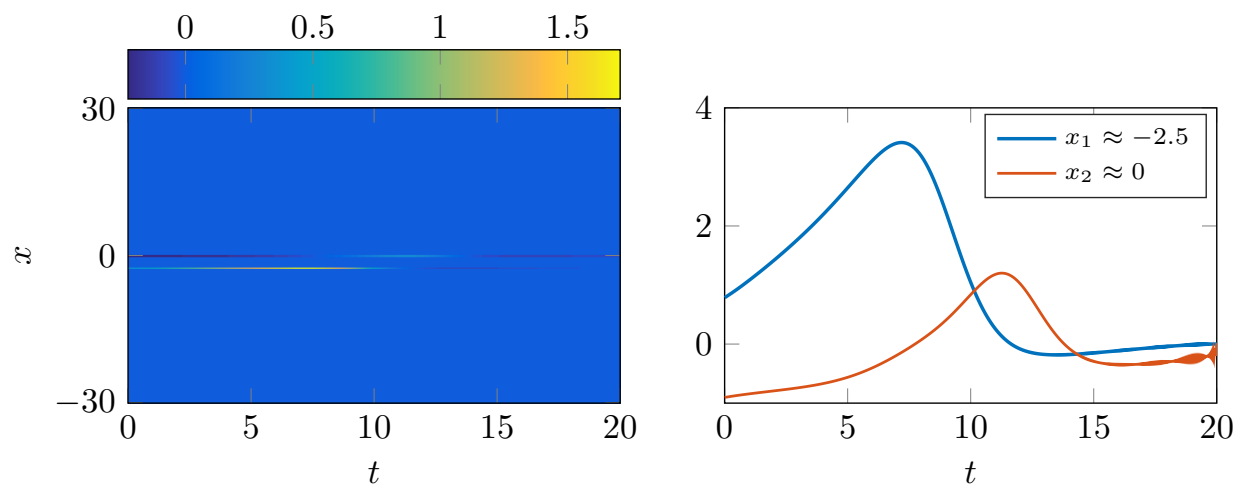

Fig. 6. Optimal control $\bar{u}, \varepsilon=10^{-5}$.

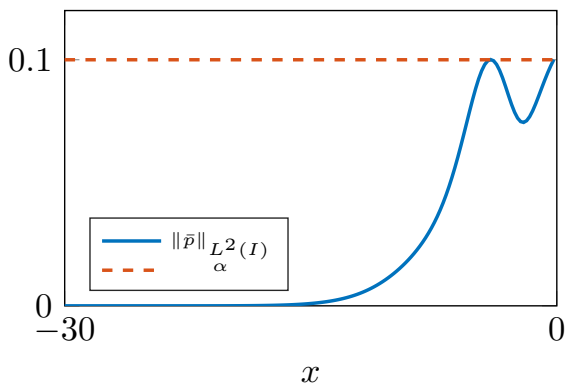

FIG. 7. Spatial support of the control determined by $\|\bar{p}(x)\|_{L^{2}(I)}=\alpha$.

the observation domain; see Figure 5 for the description of the setup. The flow enters the domain from the right and we want to create the flow profile $z$ shown in Figure 5 on $\Omega_{o}$ at final time $T$, acting only on $\Omega_{c}$. Here the space-time grid is parametrized with $N=512$ spatial grid points and a time-step size of $\Delta t=0.01$. Next, we review our numerical results. In Figure 6 the optimal control is depicted. We see that it consists of two point sources with intensities which vary smoothly in time. The $L^{2}(I)$ norm of the adjoint state $\bar{p}$ is shown in Figure 7. We clearly see that the support of the optimal control correlates with the spatial positions where $\|\bar{p}(x)\|_{L^{2}(I)}=\alpha$ holds. The optimal state $\bar{y}$ is shown in Figure 8. An upstream soliton is created that matches accurately the objective wave $z$ at terminal time. Naturally, spurious waves are created on the control domain which have no contribution to the cost functional. 

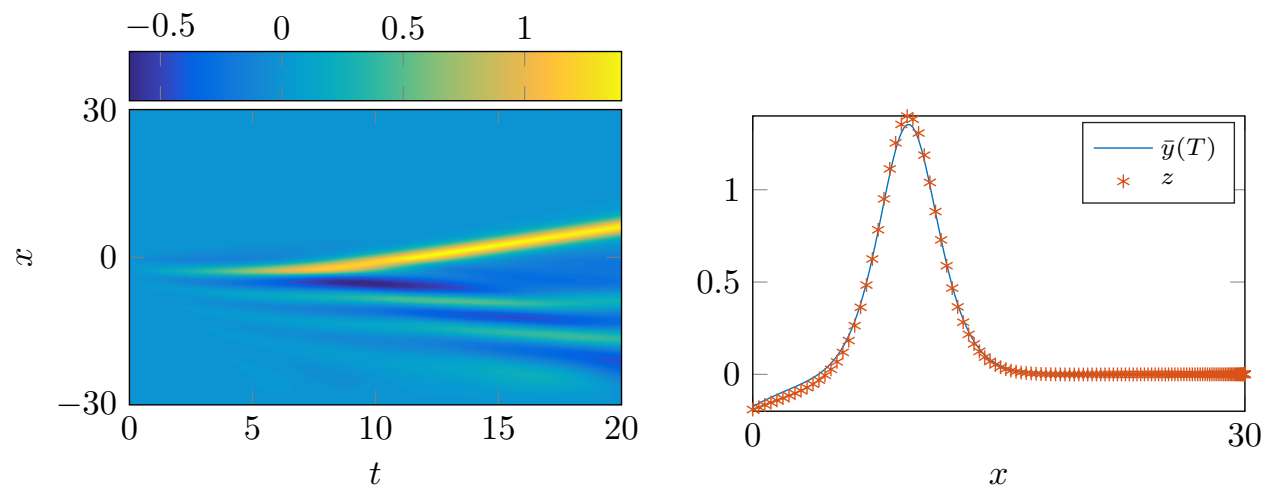

Fig. 8. Left: Evolution of the optimal state $\bar{y}$ for $\varepsilon=10^{-5}$. Right: Terminal state $\bar{y}(T)$ compared with $z$.

Appendix A. Well-posedness of the state equation, tangent equation, and adjoint equation. In this section we prove various estimates from the main part of the paper and establish the wellposedness of the linearized state equation as well as the adjoint linearized state equation.

A.1. Linear estimates: Proof of estimate (3.6). Here we consider the viscous and nonviscous case at the same time. Thus we have $\gamma \geq 0$. The proof is largely inspired from $[45,25]$. Let $y \in \mathcal{C}(\bar{I}, \mathcal{D}(A)) \cap \mathcal{C}^{1}\left(\bar{I}, L^{2}(\Omega)\right)$ be the classical solution of (3.1) for smooth versions of the data $f$ and $y_{0}$. We multiply (3.1a) which holds in $L^{2}(\Omega)$ for a.e. $t \in I$ with $y$ and get

$$
\frac{1}{2} \frac{d}{d t} \int_{0}^{L} y^{2} \mathrm{~d} x+\left|\partial_{x} y(t, 0)\right|^{2}+\gamma \int_{0}^{L}\left(\partial_{x} y\right)^{2} \mathrm{~d} x=\langle f, y\rangle_{H^{-1}(\Omega), H_{0}^{1}(\Omega)}
$$

Applying the Cauchy-Schwarz followed by Young's inequality to the right-hand side leads to

$$
\frac{1}{2} \frac{d}{d t} \int_{0}^{L} y^{2} \mathrm{~d} x+\left|\partial_{x} y(t, 0)\right|^{2}+\gamma \int_{0}^{L}\left(\partial_{x} y\right)^{2} \mathrm{~d} x \leq \frac{1}{2}\|f\|_{H^{-1}(\Omega)}^{2}+\frac{1}{2}\|y\|_{H_{0}^{1}(\Omega)}^{2} .
$$

We proceed in the same manner testing with $x y$

(A.3)

$$
\frac{1}{2} \frac{d}{d t} \int_{0}^{L} x y^{2} \mathrm{~d} x-\frac{1}{2} \int_{0}^{L} y^{2} \mathrm{~d} x+\frac{3}{2} \int_{0}^{L}\left(\partial_{x} y\right)^{2} \mathrm{~d} x+\gamma \int_{0}^{L} x\left(\partial_{x} y\right)^{2} \mathrm{~d} x=\langle f, x y\rangle_{H_{0}^{1}(\Omega), H^{-1}(\Omega)} .
$$

The right-hand side is treated using the continuity of the duality pairing, the triangle inequality, as well as Young's inequality:

(A.4)

$$
\begin{aligned}
\langle f, x y\rangle_{H^{-1}(\Omega), H_{0}^{1}(\Omega)} & \leq\|f\|_{H^{-1}(\Omega)}\left\|\partial_{x}(x y)\right\|_{L^{2}(\Omega)} \\
& \leq\|f\|_{H^{-1}(\Omega)}\left\|y+x \partial_{x} y\right\|_{L^{2}(\Omega)} \\
& \leq\|f\|_{H^{-1}(\Omega)}\left(\|y\|_{L^{2}(\Omega)}+L\left\|\partial_{x} y\right\|_{L^{2}(\Omega)}\right) \\
& \leq \frac{1}{2}\|f\|_{H^{-1}(\Omega)}^{2}+\frac{1}{2}\|y\|_{L^{2}(\Omega)}^{2}+\frac{L^{2}}{2}\|f\|_{H^{-1}(\Omega)}^{2}+\frac{1}{2}\left\|\partial_{x} y\right\|_{L^{2}(\Omega)}^{2} \\
& \leq \frac{1+L^{2}}{2}\|f\|_{H^{-1}(\Omega)}^{2}+\frac{1}{2}\|y\|_{L^{2}(\Omega)}^{2}+\frac{1}{2}\left\|\partial_{x} y\right\|_{L^{2}(\Omega)}^{2}
\end{aligned}
$$

Copyright $@$ by SIAM. Unauthorized reproduction of this article is prohibited. 
Adding (A.2), (A.3) (with the upper bound (A.4)) and omitting $\left|\partial_{x} y(t, 0)\right|^{2}$ on the left-hand side yields

$$
\frac{1}{2} \frac{d}{d t} \int_{0}^{L}(1+x) y^{2} \mathrm{~d} x+\left(\frac{1}{2}+\gamma\right)\|y\|_{H_{0}^{1}(\Omega)}^{2} \leq\left(1+\frac{L^{2}}{2}\right)\|f\|_{H^{-1}(\Omega)}^{2}+\|y\|_{L^{2}(\Omega)}^{2} .
$$

After integration between 0 and $0<t<T$ we have

$$
\begin{aligned}
\|y(t)\|_{L^{2}(\Omega)}^{2}+(1+2 \gamma) \int_{0}^{t}\|y\|_{H_{0}^{1}(\Omega)}^{2} \mathrm{~d} t \leq & \left(2+L^{2}\right) \int_{0}^{t}\|f(s)\|_{H^{-1}(\Omega)}^{2} \mathrm{~d} s \\
& +\left\|y_{0}\right\|_{L^{2}(\Omega)}^{2}+2 \int_{0}^{t}\|y(s)\|_{L^{2}(\Omega)}^{2} \mathrm{~d} s .
\end{aligned}
$$

Then the standard Gronwall inequality gives us

$$
\|y(t)\|_{L^{2}(\Omega)}^{2} \leq e^{2 t}\left(\left(2+L^{2}\right)\|f\|_{L^{2}\left(I, H^{-1}(\Omega)\right)}^{2}+\left\|y_{0}\right\|_{L^{2}(\Omega)}\right) .
$$

This yields

$$
\|y\|_{\mathcal{C}\left(\bar{I}, L^{2}(\Omega)\right)}+\|y\|_{L^{2}\left(I, H_{0}^{1}(\Omega)\right)} \leq c(T, L)\left(\|f\|_{L^{2}\left(I, H^{-1}(\Omega)\right)}+\left\|y_{0}\right\|_{L^{2}(\Omega)}\right)
$$

for some $c(T, L)>0$ independent of $y, f$, and $y_{0}$.

A.2. Nonlinear estimates: Proof of Lemma 3.6. The proof is inspired from [24, Theorem 2.8]. Let us consider $y \in \mathcal{B}$ and $z \in \mathcal{B}$. There holds $\mathcal{B} \hookrightarrow L^{4}(I \times \Omega)$ and therefore we can estimate using $\|y\|_{L^{\infty}(\Omega)}^{2} \leq c\|y\|_{L^{2}(\Omega)}\|y\|_{H_{0}^{1}(\Omega)}$

$$
\begin{gathered}
\left\|y \partial_{x} y-z \partial_{x} z\right\|_{L^{2}\left(I, H^{-1}(\Omega)\right)}=\frac{1}{2}\left(\int_{0}^{T}\left(\sup _{\|\varphi\|_{H^{1}(\Omega)}=1}\left(y^{2}-z^{2}, \partial_{x} \varphi\right)_{L^{2}(\Omega)}\right)^{2} \mathrm{~d} t\right)^{1 / 2} \\
\leq \frac{1}{2}\|z-y\|_{L^{4}(I \times \Omega)}\|z+y\|_{L^{4}(I \times \Omega)} \\
\leq \frac{1}{2}\|z-y\|_{C\left(\bar{I}, L^{2}(\Omega)\right)}^{1 / 2}\|z-y\|_{L^{2}\left(I, L^{\infty}(\Omega)\right)}^{1 / 2}\|z+y\|_{C\left(\bar{I}, L^{2}(\Omega)\right)}^{1 / 2}\|z+y\|_{L^{2}\left(I, L^{\infty}(\Omega)\right)}^{1 / 2} \\
\leq c\|z-y\|_{C\left(\bar{I}, L^{2}(\Omega)\right)}^{1 / 2}\|z+y\|_{C\left(\bar{I}, L^{2}(\Omega)\right)}^{1 / 2} \\
\left(\int_{0}^{T}\|z-y\|_{L^{2}(\Omega)}\|z-y\|_{H_{0}^{1}(\Omega)} \mathrm{d} t\right)^{1 / 4}\left(\int_{0}^{T}\|z+y\|_{L^{2}(\Omega)}\|z+y\|_{H_{0}^{1}(\Omega)} \mathrm{d} t\right)^{1 / 4} \\
\leq c T^{1 / 4}\|z-y\|_{C\left(\bar{I}, L^{2}(\Omega)\right)}^{3 / 4}\|z-y\|_{L^{2}\left(I, H_{0}^{1}(\Omega)\right)}^{1 / 4}\|z+y\|_{C\left(\bar{I}, L^{2}(\Omega)\right)}^{3 / 4}\|z+y\|_{L^{2}\left(I, H_{0}^{1}(\Omega)\right)}^{1 / 4} \\
\leq c T^{1 / 4}\|y-z\|_{\mathcal{B}}\|y+z\|_{\mathcal{B} .}
\end{gathered}
$$

A.3. The tangent equation. Next we analyze the well-posedness of the tangent equation in both the viscous and the nonviscous case, i.e., $\gamma \geq 0$.

$$
\left\{\begin{array}{l}
\partial_{t} \delta y+\partial_{x} \delta y+\partial_{x x x} \delta y-\gamma \delta \partial_{x x} y+\partial_{x}(y \delta y)=\delta u \text { in } I \times \Omega \\
\delta y(\cdot, 0)=\delta y(\cdot, L)=\partial_{x} \delta y(\cdot, L)=0 \text { in } I \\
\delta y(0, x)=\delta y_{0} \text { in } \Omega
\end{array}\right.
$$

Copyright (c) by SIAM. Unauthorized reproduction of this article is prohibited. 
Definition A.28. Let $\left(\delta u, \delta y_{0}\right) \in L^{2}\left(I, H^{-1}(\Omega)\right) \times L^{2}(\Omega)$ and $y \in \mathcal{B}$. A function $\delta y \in \mathcal{B}$ is called a solution of (A.6a)-(A.6c) if it satisfies the fixed point equation

$$
\delta y=\mathcal{L}\left(\delta u-\partial_{x}(y \delta y), \delta y_{0}\right),
$$

where $\mathcal{L}$ is the solution operator from Definition 3.4.

Proposition A.29. Let $\left(\delta u, \delta y_{0}\right) \in L^{2}\left(I, H^{-1}(\Omega)\right) \times L^{2}(\Omega)$ and $y \in \mathcal{B}$. Then, there exists a unique solution $\delta y \in \mathcal{B}$ of (A.6a)-(A.6c). Furthermore, there exists a constant $\widetilde{C}\left(T, L,\|y\|_{\mathcal{B}}\right)$ such that the following estimate holds:

$$
\|\delta y\|_{\mathcal{B}} \leq \widetilde{C}\left(\left\|\delta y_{0}\right\|_{L^{2}(\Omega)}+\|\delta u\|_{L^{2}\left(I, H^{-1}(\Omega)\right)}\right) .
$$

Proof. We define the linear mapping

$$
\Psi_{\delta u, \delta y_{0}, y}: \mathcal{B}_{\theta} \rightarrow \mathcal{B}_{\theta}, \quad \Psi_{\delta u, \delta y_{0}, y}(\delta y)=\mathcal{L}\left(\delta u-\partial_{x}(y \delta y), \delta y_{0}\right)
$$

with $\mathcal{B}_{\theta}$ defined as in (3.9) and (3.10) and $\mathcal{L}$ being the linear KdV operator described in Definition 3.4. Our goal is to show that under some constraints on $\theta, \Psi_{\delta u, \delta y_{0}, y}$ is a contraction mapping, such that the Banach fixed point theorem can be applied. First we estimate $\partial_{x}(y \delta y)$ in the $L^{2}\left((0, \theta), H^{-1}(\Omega)\right)$-norm

$$
\begin{gathered}
\text { (A.8) }\left\|\partial_{x}(y \delta y)\right\|_{L^{2}\left((0, \theta), H^{-1}(\Omega)\right)}=\left(\int_{0}^{\theta}\left(\sup _{\|v\|_{H_{0}^{1}(\Omega)}=1}\left(y \delta y, \partial_{x} v\right)_{L^{2}(\Omega)}\right)^{2} \mathrm{~d} t\right)^{1 / 2} \\
\leq\left(\int_{0}^{\theta}\|\delta y\|_{L^{2}(\Omega)}^{2}\|y\|_{L^{\infty}(\Omega)}^{2} \mathrm{~d} t\right)^{1 / 2} \leq c\|y\|_{\mathcal{C}\left([0, \theta], L^{2}(\Omega)\right)}\left(\int_{0}^{\theta}\|\delta y\|_{H_{0}^{1}(\Omega)}\|\delta y\|_{L^{2}(\Omega)} \mathrm{d} t\right)^{1 / 2} \\
\leq c \theta^{1 / 4}\|y\|_{\mathcal{C}\left([0, \theta], L^{2}(\Omega)\right)}\|\delta y\|_{\mathcal{C}\left([0, \theta], L^{2}(\Omega)\right)}^{1 / 2}\|\delta y\|_{L^{2}\left((0, \theta), H_{0}^{1}(\Omega)\right)}^{1 / 2} \\
\leq c \theta^{1 / 4}\|y\|_{\mathcal{B}_{\theta}}\|\delta y\|_{\mathcal{B}_{\theta}} .
\end{gathered}
$$

Therefore we can estimate

$$
\begin{aligned}
\left\|\Psi(\delta y)_{\delta u, \delta y_{0}, y}\right\|_{\mathcal{B}_{\theta}} & \leq \widetilde{C}\left(\left\|\delta y_{0}\right\|_{L^{2}(\Omega)}+\|\delta u\|_{L^{2}\left(I, H^{-1}(\Omega)\right)}+\left\|\partial_{x}(y \delta y)\right\|_{L^{2}\left((0, \theta), H^{-1}(\Omega)\right)}\right) \\
& \leq \widetilde{C}\left(\left\|\delta y_{0}\right\|_{L^{2}(\Omega)}+\|\delta u\|_{L^{2}\left(I, H^{-1}(\Omega)\right)}\right)+\hat{C} \theta^{1 / 4}\|y\|_{\mathcal{B}}\|\delta y\|_{\mathcal{B}_{\theta}}
\end{aligned}
$$

and

$$
\left\|\Psi_{\delta u, \delta y_{0}, y}\left(\delta y_{1}\right)-\Psi_{\delta u, \delta y_{0}, y}\left(\delta y_{2}\right)\right\|_{\mathcal{B}_{\theta}} \leq \hat{C} \theta^{1 / 4}\|y\|_{\mathcal{B}}\left\|\delta y_{1}-\delta y_{2}\right\|_{\mathcal{B}_{\theta}} .
$$

Now we set $r=3 \widetilde{C}\left(\left\|\delta y_{0}\right\|_{L^{2}(\Omega)}+\|\delta u\|_{L^{2}\left(I, H^{-1}(\Omega)\right)}\right)$ and introduce the ball

$$
B=\left\{\delta y \in \mathcal{B}_{\theta}:\|\delta y\|_{\mathcal{B}_{\theta}} \leq r\right\} .
$$

Next we choose $\theta^{*}$ small enough such that

$$
\hat{C} \theta^{* 1 / 4}\|y\|_{\mathcal{B}} \leq \frac{1}{3}
$$

holds. Then the following inequalities hold,

$$
\left\|\Psi_{\delta u, \delta y_{0}, y}(\delta y)\right\|_{\mathcal{B}_{\theta^{*}}} \leq \frac{2}{3} r, \quad\left\|\Psi_{\delta u, \delta y_{0}, y}\left(\delta y_{1}\right)-\Psi_{\delta u, \delta y_{0}, y}\left(\delta y_{2}\right)\right\|_{\mathcal{B}_{\theta^{*}}} \leq \frac{1}{3}\left\|\delta y_{1}-\delta y_{2}\right\|_{\mathcal{B}_{\theta^{*}}},
$$

Copyright (c) by SIAM. Unauthorized reproduction of this article is prohibited. 
which imply that $\Psi_{\delta u, \delta y_{0}, y}$ is a contraction mapping on $B$. So we can apply the Banach fixed point theorem which guarantees the existence of a unique fixed point $\delta y$ of $\Psi_{\delta u, \delta y_{0}, y}$ which is a solution of (A.6a)-(A.6c) in $\left(0, \theta^{*}\right)$ with initial value $\delta y_{0}$. This strategy is repeated successively using the intermediate values of $y$ as initial data. The final time $T$ can be reached since the length $\theta^{*}$ of the existence intervals is independent of the initial data; see (A.9). The concatenation of all $\delta y$ is a solution of (A.6a)-(A.6c). This strategy generates a time grid $\left(t_{k}\right)_{k=0}^{N}$ with $t_{0}=0, t_{N}=T$, and $N=\left\lceil T / \theta^{*}\right\rceil$. Next we prove the estimate (A.7). Using the time-grid we get

$$
\begin{aligned}
\|\delta y\|_{\mathcal{B}} & \leq c \sum_{k=0}^{N-1}\left(\|\delta y\|_{\mathcal{C}\left(\left[t_{k}, t_{k+1}\right], L^{2}(\Omega)\right)}+\|\delta y\|_{L^{2}\left(\left(t_{k}, t_{k+1}\right), H_{0}^{1}(\Omega)\right)}\right) \\
& \leq c \sum_{k=0}^{N-1}\left((k+1)\|\delta u\|_{L^{2}\left(I, H^{-1}(\Omega)\right)}+\left\|\delta y_{0}\right\|_{L^{2}(\Omega)}\right) \\
& \leq c\left(\frac{N(N+1)}{2}\|\delta u\|_{L^{2}\left(I, H^{-1}(\Omega)\right)}+N\left\|\delta y_{0}\right\|_{L^{2}(\Omega)}\right) .
\end{aligned}
$$

Then (A.9) implies that there exists a $c>0$ such that

$$
N \leq \frac{T}{\theta^{*}}+1=c T\|y\|_{\mathcal{B}}^{4}+1
$$

and accordingly

$$
\|\delta y\|_{\mathcal{B}} \leq c\left(\|y\|_{\mathcal{B}}\right)\left(\|\delta u\|_{L^{2}\left(I, H^{-1}(\Omega)\right)}+\left\|\delta y_{0}\right\|_{L^{2}(\Omega)}\right) .
$$

Finally we discuss uniqueness. Let $\delta y \in \mathcal{B}$ be a solution of (A.6a)-(A.6c) for $\delta u=$ $\delta y_{0}=0$. From (A.5) we see

$$
\begin{aligned}
\|\delta y(t)\|_{L^{2}(\Omega)}^{2} & \leq c \int_{0}^{t}\left\|\partial_{x}(y \delta y)(s)\right\|_{H^{-1}(\Omega)}^{2}+\|\delta y(s)\|_{L^{2}(\Omega)}^{2} \mathrm{~d} s \\
& \leq \tilde{c} \int_{0}^{t}\left(\|y(s)\|_{H_{0}^{1}(\Omega)}^{2}+1\right)\|\delta y(s)\|_{L^{2}(\Omega)}^{2} \mathrm{~d} s
\end{aligned}
$$

for any $T \geq t \geq 0$. Then Gronwall's lemma implies $y \equiv 0$. Thus existence of a unique solution is proved.

A.4. The adjoint equation. Next we study the equation

$$
\left\{\begin{array}{l}
-\partial_{t} p-\partial_{x} p-\partial_{x x x} p-\gamma \partial_{x x} p-y \partial_{x} p=\phi \text { in } I \times \Omega \\
p(\cdot, 0)=p(\cdot, L)=\partial_{x} p(\cdot, 0)=0 \text { on } I \\
p(T)=p_{T} \text { in } \Omega
\end{array}\right.
$$

for any $y \in \mathcal{B}$ and $\gamma \geq 0$, i.e., in the viscous and nonviscous case.

Definition A.30. A function $p \in \mathcal{B}$ is called a solution of (A.10a)-(A.10c) if it solves the fixed point equation

$$
p(t)=W^{*}(t) p_{T}+\int_{t}^{T} W^{*}(s-t)\left(\phi(s)+y(s) \partial_{x} p(s)\right) \mathrm{d} s .
$$

Lemma A.31. Let $y \in \mathcal{B}, p \in \mathcal{B}$. Then it holds that

$$
\left\|y \partial_{x} p\right\|_{L^{1}\left(0, T, L^{2}(\Omega)\right)} \leq c T^{1 / 4}\|y\|_{\mathcal{B}}\|p\|_{\mathcal{B}}
$$

Copyright (C) by SIAM. Unauthorized reproduction of this article is prohibited. 
Proof. We estimate

$$
\begin{gathered}
\left\|y \partial_{x} p\right\|_{L^{1}\left(I, L^{2}(\Omega)\right)} \leq \int_{0}^{T}\|y\|_{L^{\infty}(\Omega)}\|p\|_{H_{0}^{1}(\Omega)} \mathrm{d} t \\
\leq c \int_{0}^{T}\|y\|_{L^{2}(\Omega)}^{1 / 2}\|y\|_{H_{0}^{1}(\Omega)}^{1 / 2}\|p\|_{H_{0}^{1}(\Omega)} \mathrm{d} t \\
\leq c\|y\|_{C\left(\bar{I}, L^{2}(\Omega)\right)}^{1 / 2}\left(\int_{0}^{T}\|y\|_{H_{0}^{1}(\Omega)} \mathrm{d} t\right)^{1 / 2}\|p\|_{L^{2}\left(I, H_{0}^{1}(\Omega)\right)} \\
\quad \leq c T^{1 / 4}\|y\|_{C\left(\bar{I}, L^{2}(\Omega)\right)}^{1 / 2}\|y\|_{L^{2}\left(I, H_{0}^{1}(\Omega)\right)}^{1 / 2}\|p\|_{L^{2}\left(I, H_{0}^{1}(\Omega)\right)},
\end{gathered}
$$

which implies the assertion.

Proposition A.32. Let $\left(\phi, p_{T}\right) \in L^{1}\left(I, L^{2}(\Omega)\right) \times L^{2}(\Omega)$. Then (A.10a)-(A.10c) has a unique solution $p \in \mathcal{B}$. Furthermore there exists a constant $c\left(\|y\|_{\mathcal{B}}\right)>0$ such that

$$
\|p\|_{\mathcal{B}} \leq c\left(\left\|p_{T}\right\|_{L^{2}(\Omega)}+\|\phi\|_{L^{1}\left(I, L^{2}(\Omega)\right)}\right)
$$

holds.

Proof. First we change the variables in the adjoint equation by using the transformation $\tilde{x}=L-x$ and $\tilde{t}=T-t$. Then uniqueness follows from [20, Proposition 15]. Existence and the estimate (A.11) are proved in the same way as in the proof of Proposition A.29. Hereby we rely on Lemma A.31.

\section{REFERENCES}

[1] D. N. Arnold And R. Winther, A superconvergent finite element method for the Korteweg-de Vries equation, Math. Comp., 38 (1982), pp. 23-36.

[2] H. H. Bauschke and P. L. Combettes, Convex Analysis and Monotone Operator Theory in Hilbert Spaces, Springer, New York, 2011.

[3] A. Bensoussan, G. Da Prato, M. C. Delfour, and S. K. Mitter, Representation and Control of Infinite Dimensional Systems, 2nd ed., Systems Control Found. Appl., Birkhäuser, Boston, 2007, https://doi.org/10.1007/978-0-8176-4581-6.

[4] J. Bona AND R. WInTHER, The Korteweg-de Vries equation posed in a quarter-plane, SIAM J. Math. Anal., 14 (1983), pp. 1056-1106, https://doi.org/10.1137/0514085.

[5] J. L. Bona, V. A. Dougalis, and O. A. Karakashian, Fully discrete galerkin methods for the Korteweg-de Vries equation, Comput. Math. Appl., 12 (1986), pp. 859-884.

[6] J. L. Bona, S.-M. Sun, AND B.-Y. Zhang, A nonhomogeneous boundary-value problem for the Korteweg-de Vries equation posed on a finite domain, Comm. Partial Differential Equations, 28 (2003), pp. 1391-1436, https://doi.org/10.1081/PDE-120024373.

[7] J. Bourgain, Fourier transform restriction phenomena for certain lattice subsets and applications to nonlinear evolution equations. II. The KdV-equation, Geom. Funct. Anal., 3 (1993), pp. 209-262, https://doi.org/10.1007/BF01895688.

[8] J. Bourgain, Periodic Korteweg-de Vries equation with measures as initial data, Selecta Math. (N.S.), 3 (1997), pp. 115-159.

[9] H. Brezis, Functional Analysis, Sobolev Spaces and Partial Differential Equations, Universitext, Springer, New York, 2011.

[10] E. Casas, C. Clason, And K. Kunisch, Approximation of elliptic control problems in measure spaces with sparse solutions, SIAM J. Control Optim., 50 (2012), pp. 1735-1752.

[11] E. Casas, C. Clason, and K. Kunisch, Parabolic control problems in measure spaces with sparse solutions, SIAM J. Control Optim., 51 (2013), pp. 28-63.

[12] E. CASAS AND K. KUnisch, Parabolic control problems in space-time measure spaces, ESAIM Control Optim. Calc. Var., 22 (2016), pp. 355-370.

[13] E. Casas, B. Vexler, and E. Zuazua, Sparse initial data indentification for parabolic PDE and its finite element approximations, Math. Control Relat. Fields, 5 (2015), pp. 377-399, https://doi.org/10.3934/mcrf.2015.5.377.

Copyright $@$ by SIAM. Unauthorized reproduction of this article is prohibited. 
[14] E. Casas and E. Zuazua, Spike controls for elliptic and parabolic PDEs, Systems Control Lett., 62 (2013), pp. 311-318, https://doi.org/10.1016/j.sysconle.2013.01.001.

[15] M. Chapouly, Global controllability of a nonlinear Korteweg-de Vries equation, Commun. Contemp. Math., 11 (2009), pp. 495-521.

[16] C. Clason and B. Kaltenbacher, On the use of state constraints in optimal control of singular PDEs, Systems Control Lett., 62 (2013), pp. 48-54, https://doi.org/10.1016/j. sysconle.2012.10.006.

[17] C. Clason and K. Kunisch, A duality-based approach to elliptic control problems in nonreflexive banach spaces, ESAIM Control Optim. Calc. Var., 17 (2011), pp. 243-266.

[18] C. Clason and K. KUnisch, A measure space approach to optimal source placement, Comput. Optim. Appl., 53 (2011), pp. 155-171.

[19] A. Constantin AND R. Johnson, On the non-dimensionalisation, scaling and resulting interpretation of the classical governing equations for water waves, J. Nonlinear Math. Phys., 15 (2008), pp. 58-73.

[20] J.-M. CORON AND E. CRÉPEAu, Exact boundary controllability of a nonlinear Korteweg-de Vries equation with critical lengths, J. Eur. Math. Soc. (JEMS), 6 (2004), pp. 367-398.

[21] K. Duidjeli, W. G. Price, E. H. Twizell, And Y. Wang, Numerical methods for the solution of the third-and fifth-order dispersive Korteweg-de Vries equations, J. Comput. Appl. Math., 58 (1995), pp. 307-336.

[22] D. Dutykh, T. Katsaounis, and D. Mitsotakis, Finite volume methods for unidirectional dispersive wave models, Internat. J. Numer. Methods Fluids, 71 (2013), pp. 717-736.

[23] I. Ekeland and R. TÉmam, Convex Analysis and Variational Problems, Classics Appl. Math. 28, SIAM, Philadelphia, 1999, https://doi.org/10.1137/1.9781611971088 (in English).

[24] A. V. FAminskit AND N. A. LARKIn, Initial-boundary value problems for quasilinear dispersive equations posed on a bounded interval, Electron. J. Differential Equations, 2010 (2010), pp. $1-20$.

[25] O. Glass and S. Guerrero, Some exact controllability results for the linear Korteweg-de Vries equation and uniform controllability in the zero-dispersion limit, Asymptot. Anal., 60 (2008), pp. 61-100.

[26] W. Hensgen, A simple proof of Singer's representation theorem, Proc. Amer. Math. Soc., 124 (1996), pp. 3211-3212, https://doi.org/10.1090/S0002-9939-96-03493-4.

[27] R. Herzog, G. Stadler, and G. Wachsmuth, Directional sparsity in optimal control of partial differential equations, SIAM J. Control Optim., 50 (2012), pp. 943-963.

[28] C. E. Kenig, G. Ponce, And L. Vega, Well-posedness and scattering results for the generalized Korteweg-de Vries equation via the contraction principle, Comm. Pure Appl. Math., 46 (1993), pp. 527-620, https://doi.org/10.1002/cpa.3160460405.

[29] D. J. Korteweg And G. De VRIes, On the change of form of long waves advancing in a rectangular canal, and on a new type of long stationary waves, London Edinburgh Dublin Philos. Mag. J. Sci., 39 (1895), pp. 422-443.

[30] K. Kunisch, K. Pieper, AND A. RUND, Time optimal control for a reaction diffusion system arising in cardiac electrophysiology - A monolithic approach, ESAIM Math. Model. Numer. Anal., 50 (2016), pp. 381-414.

[31] K. Kunisch, K. Pieper, And B. Vexler, Measure valued directional sparsity for parabolic optimal control problems, SIAM J. Control Optim., 52 (2014), pp. 3078-3108, https://doi. org $/ 10.1137 / 140959055$.

[32] K. Kunisch, P. Trautmann, And B. Vexler, Optimal control of the undamped linear wave equation with measure valued controls, SIAM J. Control Optim., 54 (2016), pp. 1212-1244.

[33] S. LAng, Real Analysis, 2nd ed., Addison-Wesley, Reading, MA, 1983.

[34] J. LI, H. MA, AND W. Sun, Error analysis for solving the Korteweg-de Vries equation by a Legendre pseudo-spectral method, Numer. Methods Partial Differential Equations, 16 (2000), pp. 513-534.

[35] J.-L. Lions, Contrôle des systèmes distribués singuliers, Math. Methods Inform. Sci. 13, Gauthier-Villars, Montrouge, 1983.

[36] H. Ma And W. Sun, A Legendre-Petrov-Galerkin and Chebyshev collocation method for thirdorder differential equations, SIAM J. Numer. Anal., 38 (2000), pp. 1425-1438.

[37] H. Ma And W. Sun, Optimal error estimates of the Legendre-Petrov-Galerkin method for the Korteweg-de Vries equation, SIAM J. Numer. Anal., 39 (2001), pp. 1380-1394.

[38] D. Meidner, Adaptive Space-Time Finite Element Methods for Optimization Problems Governed by Nonlinear Parabolic Systems, Ph.D. thesis, Universität Heidelberg, 2007.

[39] P. A. Milewski, The forced Korteweg-de Vries equation as a model for waves generated by topography, CUBO, 6 (2004), pp. 33-51.

Copyright $@$ by SIAM. Unauthorized reproduction of this article is prohibited. 
[40] R. M. Miura, The Korteweg-de Vries equation: A survey of results, SIAM Rev., 18 (1976), pp. 412-459.

[41] H. Nersisyan, D. Dutykh, And E. Zuazua, Generation of $2 D$ water waves by moving bottom disturbances, IMA J. Appl. Math., 80 (2015), pp. 1235-1253.

[42] A. PAzy, Semigroups of Linear Operators and Applications to Partial Differential Equations, Vol. 198, Springer, New York, 1983.

[43] K. Pieper, Finite Element Discretization and Efficient Numerical Solution of Elliptic and Parabolic Sparse Control Problems, Ph.D. thesis, Technische Unisversität München, 2015.

[44] K. Pieper and B. Vexler, A priori error analysis for discretization of sparse elliptic optimal control problems in measure space, SIAM J. Control Optim., 51 (2013), pp. 2788-2808.

[45] L. Rosier, Exact boundary controllability for the Korteweg-de Vries equation on a bounded domain, ESAIM Control Optim. Calc. Var., 2 (1997), pp. 33-55.

[46] J. Shen, A new dual-Petrov-Galerkin method for third and higher odd-order differential equations: Application to the KdV equation, SIAM J. Numer. Anal., 41 (2003), pp. 1595-1619.

[47] S. S.-P. ShEn, Forced solitary waves and hydraulic falls in two-layer flows, J. Fluid Mech., 234 (1992), pp. 583-612.

[48] S. S.-P. SHEn, On the accuracy of the stationary forced Korteweg-de Vries equation as a model equation for flows over a bump, Quart. Appl. Math., 53 (1996), pp. 701-720.

[49] R. E. Showalter, Monotone Operators in Banach Space and Nonlinear Partial Differential Equations, Math. Surveys Monogr. 49, AMS, Providence, RI, 1997.

[50] T. Steinaug, The conjugate gradient method and trust regions in large scale optimization, SIAM J. Numer. Anal., 20 (1983), pp. 626-637, https://doi.org/10.1137/0720042.

[51] C. Su And C. Gardner, Korteweg-de Vries equation and generalizations. III. Derivation of the Korteweg-de Vries equation and Burgers equation, J. Math. Phys., 10 (2003), pp. 536-539.

[52] M. UlBRICH, Semismooth Newton methods for operator equations in function spaces, SIAM J. Optim., 13 (2002), pp. 805-841.

[53] R. Winther, A conservative finite element method for the Korteweg-de Vries equation, Math. Comp., 34 (1980), pp. 23-43.

[54] J. YAN AND C.-W. ShU, A local discontinuous Galerkin method for KdV type equations, SIAM J. Numer. Anal., 40 (2002), pp. 769-791.

[55] N. J. ZABUSKY AND M. D. KRUSKAL, Interaction of solitons in a collisionless plasma and the recurrence of initial states, Phys. Rev. Lett, 15 (1965), pp. 240-243.

Copyright $@$ by SIAM. Unauthorized reproduction of this article is prohibited. 